\title{
Spin and Spin-Wave Dynamics in Josephson Junctions
}

\author{
Zohar Nussinov ${ }^{1,2}$, Alexander Shnirman ${ }^{3}$, Daniel P. Arovas ${ }^{4}$, Alexander V. Balatsky ${ }^{1}$, and Jian Xin Zhu ${ }^{1}$ \\ ${ }^{1}$ Theoretical Division, Los Alamos National Laboratory, Los Alamos, NM 87545, USA \\ ${ }^{2}$ Department of Physics, Washington University, St. Louis, MO 63160-4899, USA \\ ${ }^{3}$ Institut für Theoretische Festkörperphysik, Universität Karlsruhe, D-76128 Karlsruhe, Germany and \\ ${ }^{4}$ Department of Physics, University of California, San-Diego, La Jolla, CA 92093, USA
}

(Dated: Received February 2, 2008)

\begin{abstract}
We extend the Keldysh formulation to quantum spin systems and derive exact equations of motion. This allows us to explore the dynamics of single spins and of ferromagnets when these are inserted between superconducting leads. Several new effects are reported. Chief amongst these are nutations of single $S=1 / 2$ spins in Josephson junctions. These nutations are triggered by the superconducting pairing correlations in the leads. Similarly, we find that on rather universal grounds, magnets display unconventional spin wave dynamics when placed in Josephson junctions. These lead to modifications in the tunneling current.

PACS numbers: 71.27.+a, 71.28.+d, 77.80.-e
\end{abstract}

\section{INTRODUCTION}

There is a growing interest in a number of techniques that allow detection and manipulation of a single spin. A partial list includes optical detection of electron spin resonance (ESR) in a single molecule [1], tunneling through a quantum dot [2], and, more recently, the ESR-scanning tunneling microscopy (ESR-STM) technique [3, 4]. Interest in ESRSTM lies in the potential of detection and manipulation of a single spin [5, 6]- an ability which is crucial to spintronics and quantum information processing. Much work also addressed coupling, feedback effects, and decoherence in a coupled electronic-vibrational systems, such as nanomechanical oscillators and local vibrational modes [7]. In particular, spintronic and quantum computing applications greatly intensified interest in Josephson junctions. In a previous publication [8], four of us studied the effect of the supercurrent on a macroscopic spin cluster (of spin $S \gg 1$ ) precessing in the presence of a magnetic field when placed in a Josephson junction to find new spin dynamics. In [9], these systems were examined anew wherein variations in the DC current were predicted for all systems harboring a spin of any finite size $S$. In the current article, we complement [8] by studying, for the first time, the explicit dynamics of single quantum $S=1 / 2$ spins in Josephson junctions to find new intriguing dynamical effects for which we provide quantitative expressions. The single spin $(S=1 / 2)$ dynamics which we study here differs significantly from the the large magnetic cluster $(S \gg 1)$ dynamics studied in [8]. In the current article, we further examine spin wave dynamics in ferromagnets when placed in Josephson junctions.

The analysis of spins embedded in Josephson junctions has a long and rich history. Early on, Kulik [10] argued that spin flip processes in tunnel barriers reduce the critical Josephson current as compared to the Ambegaokar-Baratoff limit [11]. More than a decade later, Bulaevskii et al. [12] conjectured that $\pi$-junctions may form if spin flip processes dominate. The competition between the Kondo effect and the superconductivity was elucidated in [13]. A nice review of experimental works on certain aspects of magnetic nanoparticles in Josephson junctions is found in [14]. Transport properties formed the central core of many pioneering works, while spin dynamics was relegated a relatively trivial secondary role. In the current article, we report on exact new non-stationary spin dynamics and illustrate how a quantum $S=1 / 2$ spin is affected by the Josephson current. As a consequence of the Josephson current, spins exhibit non-planar precessions while subject to the external magnetic field. As well known, a single quantum spin in a magnetic field exhibits circular Larmor precession about the direction of the field. As we report here, when the spin is further embedded between two superconducting leads, quantum pairing correlations lead to new out-of-plane longitudinal motion, much alike that displayed by a classical mechanical top will arise. We term this effect the Josephson nutation. Similar effects occur when a ferromagnetic slab is placed between two superconducting leads. We outline how transport is, in turn, modulated by this rather unusual spin dynamics. The coupling of the spin to the supercurrent leads to an effective non-local in time interaction of the single spin with itself. Keldysh contour calculations illustrate that a non-local in time single fermion action is also found in situations wherein the single spin is replaced by an Anderson impurity [15]. As well known, in the limit of small hopping amplitudes to and from an Anderson impurity, the impurity attains a Kondo like character much like that of the single spin which is the focus of our attention. Here we consider the origin of this rather generic non-locality in time present in the dynamics of a Josephson junction. En route to deriving this new spin dynamics we illustrate that even in the presence of non-local in time interactions, certain variants of the classical equations of motion become trivially exact by virtue of compactness of the spin variables. An elaborate extension of these ideas will be detailed elsewhere [16].

\section{OUTLINE OF THE ARTICLE}

The main goal of the current publication is to report on the spin and spin wave dynamics (of single spins and of magnetic systems, respectively) in Josephson junctions.

To achieve this aim, we will initially (in Sections(IIIIV) extend the non-equilibrium Keldysh formalism to address 
these problems. In Section(III), we illustrate that even in the presence of effective non-local of time interactions of a spin with itself (such as those borne by the interaction of a single spin with a Josephson current), the equations of motion undergo a trivial modification. In Section IV, we rewrite these equations within the standard Keldysh basis best suited for non-equilibrium problems. Sections III IV may be seen as independent extensions of basic facets of the non-equilibrium Keldysh formalism for a spin system.

In Section( $\mathrm{V}$, we apply the rather general formalism developed in Sections (IIIIV to the specific problem of a single $S=1 / 2$ spin in a Josephson junction (with a time independent potential difference between the two superconducting leads). We start, in subsection $\mathrm{VA}$, in writing down the relevant Hamiltonian of such a Josephson junction harboring a single spin. In subsection $(\nabla \mathrm{B}$, we briefly highlight the natural time scales in the problem- which will indeed come to the fore in the detailed solution which we will later expose. In the all-important subsection V $\mathrm{C}$, we highlight the origin of the effective non-local in time interactions of the spin with itself. Here, we integrate out the lead electrons to find the effective spin only action harboring such nonlocal in time interactions. These non-trivial interactions are the reason that we needed to develop and extend (Sections (IIIIV) the Keldysh formalism to a very general spin system with such interactions. In subsection VD, we invoke the results of Sections IIIIV to the resultant effective spinonly action of Section $\mathrm{VC}$ to write down the equations of motion for the spin. In subsection $(\mathrm{VE})$, we solve these equations of motion to lowest order in the spin-dependent tunneling amplitude. Detailed technical aspects of the solution on which subsection $\mathrm{VE}$ dutifully relies on have been relegated to appendices $\mathrm{B}$ and $\mathrm{C}$. The perturbative solution to the equations of motion- the final equations of subsection( $\nabla E$ )- form one of the main core results of the current publication. In subsection $\mathrm{VF}$, we examine the physical meaning of this solution of the single spin problem to unearth several new predictions for this $S=1 / 2$ system. In this subsection, we aim to further arm the reader with an intuitive understanding for the physical origin of these new effects. Some of these predicted effects (and our prediction of nutation in particular) are highlighted in Fig. (3). In subsection(DI), we examine the behavior of the system for a single spin of magnitude $S>1 / 2$. In the large $S \gg 1$ limit, we recover our very different semiclassical spin $(S \rightarrow \infty)$ results of [8].

Next, in Section (VII), we discuss a variation of the single spin problem wherein an $\mathrm{AC}$ voltage bias is applied across the Josephson junction. Our main result are the predictions of specific time dependent spin dynamics displaying an infinite number of harmonics and new DC lock-in effects. The predicted supercurrent in this system is also discussed.

In Section (VIII), we examine the problem of a ferromagnet in a Josephson junction. In the spin-wave approximation, we find that each spin-wave mode displays some of the unusual effects predicted in subsection $\mathrm{VE} \mathrm{VF}$ for the single spin problem. The predicted spin wave dynamics and associated transport (current), are furnished. In Section(IX), we discuss simple extensions of our results to other systems generated by

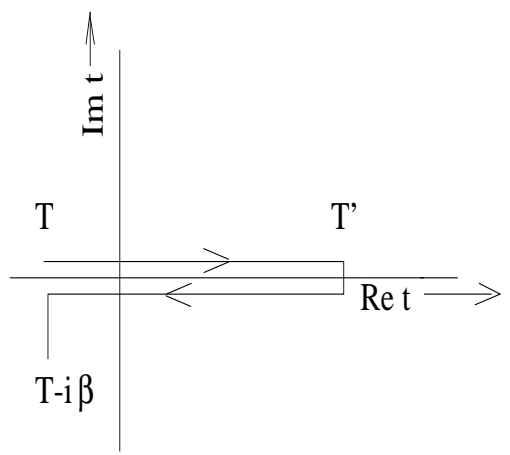

FIG. 1: The standard Keldysh contour. The times $T$ and $T^{\prime}$ are taken to be $-\infty$ and $\infty$ respectively. The form of this contour will be heavily employed in our work when time ordering various spin products.

a trivial change of geometry wherein at least one of the superconductors forming the Josephson junction is replaced by a planar superconductor. In Section $\mathrm{X}$, we write down the $S \gg 1$ equations of motion for general magnets and antiferromagnetic chains. The non-uniform temporal evolution of each of the spin-waves is highlighted in the resultant solution. We conclude the main text, in Section XI , by highlighting our conclusions.

In Appendix A, we briefly discuss several experimental manifestations of our effect and highlight a proposed experiment which may verify our predictions.

\section{EXACT SPIN-1/2 EQUATIONS OF MOTION ON KELDYSH CONTOURS}

We start by deriving the equations of motion for a very general spin-1/2 system having two (or more) local and nonlocal spin-spin interactions at different times. In this work we employ the non-equilibrium Keldysh technique. Within this framework, the spin operators on both up and down portions of the (Keldysh) contour of Fig. 1 are normalized and satisfy $\left[\vec{S}_{u}(t), \vec{S}_{d}\left(t^{\prime}\right)\right]=0$. In what briefly follows, we will dispense with operator formulations and employ a path integral representation. Towards this end, our working horses will be the $C P_{1}$ spin coherent variables $(z)[17,18]$ wherein the spins are represented by

$$
\vec{S}=S z_{a}^{*} \vec{\sigma}_{a b} z_{b}
$$

(with $S$ the spin magnitude). Here and throughout, we set $\hbar=$ 1. In Eq. (1), $a, b=\uparrow, \downarrow$ and we assume an implied summation over repeated indices. The vectors $\vec{\sigma}_{a b}$ are the $a b$ components of the three Pauli matrices. The components $z_{a=\uparrow, \downarrow}$ code for a two component complex spinor subject to the normalization constraint, $\left|z_{\uparrow}\right|^{2}+\left|z_{\downarrow}\right|^{2}=1$. By glancing at Eq. 1), we note that a knowledge of $\vec{S}$ specifies the two component spinor $z$ only up to a global multiplicative phase.

As well appreciated, in a spin coherent basis, the Berry phases associated with the spin coherent states are the net area of the spherical triangle spanned by the spin as it moves on the Bloch sphere. The latter may be expressed in the $C P_{1}$ basis as 
$S_{\text {Berry }}=i \int d t \sum_{a} z_{a}^{*} \partial_{t} z_{a}[17,18]$. For the benefit of readers unfamiliar with this formalism, we provide in [19] a quick derivation for this form of the Berry phase.

We now assume the action contains the single spin term $-\eta_{a} \int d t \vec{S}_{a} \cdot \vec{h}$ describing a single spin in an external magnetic field set by $\vec{h}$. The parity $\eta_{a}= \pm 1$ is fixed by the direction of the contour- $\eta_{u p}=1, \eta_{\text {down }}=-1$. We further include a nonlocal in time spin interaction $\eta_{a} \eta_{b} \int d t \int d t^{\prime} \bar{K}_{a b}\left(t, t^{\prime}\right) \vec{S}_{a}(t)$. $\vec{S}_{b}\left(t^{\prime}\right)$. The kernels $\bar{K}_{a b}$ encapsulate non-local temporal dependence. The generalization to higher order terms is straightforward and leads to no qualitative change. With the Berry phase included, the general action

$$
\begin{array}{r}
S=2 i S \eta_{a} \int d t z_{a}^{*} \partial_{t} z_{a}-S \int d t \eta_{a} \vec{h}_{a} \cdot z_{a}^{*} \vec{\sigma} z_{a} \\
+S^{2} \eta_{a} \eta_{b} \int d t \int d t^{\prime} \bar{K}_{a b}\left(t, t^{\prime}\right) z_{a}^{*}(t) \vec{\sigma} z_{a}(t) \cdot z_{b}^{*}\left(t^{\prime}\right) \vec{\sigma} z_{b}\left(t^{\prime}\right) .
\end{array}
$$

Varying the action,

$$
\begin{array}{r}
\frac{\delta S}{\delta z_{a \nu}^{*}(t)}=S \eta_{a}\left(2 i \partial_{t} z_{a \nu}(t)-\vec{h} \cdot \vec{\sigma}_{\nu \gamma} z_{a \gamma}(t)\right. \\
\left.+\eta_{b} \int d t^{\prime} K_{a b}\left(t, t^{\prime}\right) z_{b \gamma}^{*}\left(t^{\prime}\right) \vec{\sigma}_{\gamma \delta} z_{b \delta}\left(t^{\prime}\right) \cdot \vec{\sigma}_{\nu \gamma} z_{a \gamma}(t)\right) \\
\equiv S \eta_{a}\left(2 i \partial_{t} z_{a \nu}(t)-\vec{H}(t) \cdot \vec{\sigma}_{\nu \gamma} z_{a \gamma}(t)\right) .
\end{array}
$$

Here,

$$
\begin{array}{r}
K_{a b}\left(t, t^{\prime}\right) \equiv \bar{K}_{a b}\left(t, t^{\prime}\right)+\bar{K}_{b a}\left(t^{\prime}, t\right) \\
\vec{H}(t) \equiv \vec{h}+S \eta_{a} \int d t^{\prime} K_{a b}\left(t, t^{\prime}\right) z_{b \gamma}^{*}\left(t^{\prime}\right) \vec{\sigma}_{\gamma \delta} z_{b \delta}\left(t^{\prime}\right) .
\end{array}
$$

Next, we briefly generalize Ehrenfest's theorem to situations such as the one of relevance here where a non-local in time action is present. A full discussion of this theorem for general systems will be presented elsewhere [16]. In what follows, the expectation value of any quantity $\mathcal{A}$ evaluated with the action $S$ is denoted by

$$
\langle\mathcal{A}\rangle_{S} \equiv \frac{1}{Z} \int D z D z^{*} \delta\left(|z|^{2}-1\right) \mathcal{A} e^{i S},
$$

with $Z=\int D z D z^{*} \delta\left(|z|^{2}-1\right) e^{i S}$ the associated partition function. Similar definitions apply, with a trivial replacement of the measure when the action is a functional of one of more real fields $\left\{x_{\alpha}(t)\right\}$. In the current context, $x_{\alpha}$ code for the real or imaginary parts of the complex spinor components $z$. Next, we note that for any cyclic coordinate $x$, the expectation value of the variational derivative,

$$
\left\langle\frac{\delta S}{\delta x}\right\rangle_{S}=\frac{-i}{Z}\left[e^{i S}\right]_{x_{i}(t)}^{x_{f}(t)}=0 .
$$

In the above, by the compactness of $x$, in integrating all possible trajectories $x(t)$, the initial and final trajectories are equal $x_{i}(t)=x_{f}(t)$. This in turn lead to the vanishing expectation value given in Eq. (6) for all non-singular actions. Analogously, this result follows by noting that for compact coordinates, the transformation $[x(t) \rightarrow x(t)+\delta x(t)]$, with any $\delta x(t)$ leads to no change to the value of $Z$ - the range of integration in $Z=\int D x e^{i S}$ is unchanged. This, in turn, mandates that $\left\langle\frac{\delta S}{\delta x}\right\rangle_{S}=0$ [16]. Next, we consider $\mathcal{A}^{i} \equiv z^{*} \sigma^{i} \frac{\delta S}{\delta z^{*}}$ and explicitly illustrate that its expectation value vanishes, $\left\langle\mathcal{A}^{i}\right\rangle=0$. To this end, we write the spinors, longhand, in terms of real and imaginary components, $z^{*}=$ $\left(z_{R e}^{1}-i z_{I m}^{1} z_{R e}^{2}-i z_{I m}^{2}\right)$ and the measure $D z D z^{*} \delta\left(|z|^{2}-1\right)=$ $D z_{R e}^{1} D z_{I m}^{1} D z_{R e}^{2} D z_{I m}^{2} \delta\left(\left|z_{R e}^{1}\right|^{2}+\left|z_{I m}^{1}\right|^{2}+\left|z_{R e}^{2}\right|^{2}+\left|z_{I m}^{2}\right|^{2}-\right.$ $1)$. Here and in what briefly follows we suppress a uniform Keldysh contour index. The expectation value $\left\langle A^{i}\right\rangle$ for each value of the spin index, $i=x, y, z$, is an integral over bilinears in $z$ and hence amounts to a sum of integrals of the type

$$
\begin{array}{r}
I_{\alpha \beta} \equiv \int D z_{R e}^{1} D z_{I m}^{1} D z_{R e}^{2} D z_{I m}^{2} \\
\delta\left(\left|z_{R e}^{1}\right|^{2}+\left|z_{I m}^{1}\right|^{2}+\left|z_{R e}^{2}\right|^{2}+\left|z_{I m}^{2}\right|^{2}-1\right) z_{\alpha} \frac{\delta S}{\delta z_{\beta}} e^{i S} .
\end{array}
$$

Here, the indices $\alpha$ and $\beta$ span the four possible values (1 Re, $1 \mathrm{Im}, 2 \mathrm{Re}, 2 \mathrm{Im})$. An immediate consequence of the vanishing of the expectation value $\left\langle\frac{\delta S}{\delta x}\right\rangle$ for any cyclic coordinate $x$ is that all integrals of the form $I_{\alpha \neq \beta}$ vanish. An inspection of $\left\langle\mathcal{A}^{i}\right\rangle$ reveals that the contributions of all integrals of the type $I_{\alpha \alpha}$ cancel identically when $i=z$ (the only place where integrals of the type $I_{\alpha=\beta}$ appear). Similarly, $\left\langle\mathcal{A}^{i \dagger}\right\rangle=\left\langle\frac{\delta S}{\delta z(t)} \sigma^{i} z\right\rangle=0$. The vanishing $\left\langle\mathcal{A}^{i}\right\rangle=\left\langle\mathcal{A}^{i \dagger}\right\rangle=0$ imply that their difference,

$$
0=\left\langle\left[\frac{\delta S}{\delta z_{a \sigma}(t)} \sigma_{\sigma \sigma^{\prime}}^{j} z^{a \sigma^{\prime}}-z_{a \sigma}^{*} \sigma_{\sigma \sigma^{\prime}}^{j} \frac{\delta S}{\delta z_{a \sigma^{\prime}}^{*}(t)}\right]\right\rangle_{S},
$$

where the Keldysh contour index $(a)$ is reinstated.

Next, we explicitly insert Eq. (3) into Eq.8. As a consequence of the $\mathrm{SU}(2)$ algebra of the Pauli matrices, we find that for each Keldysh contour index $a=$ top/bottom,

$$
\left\langle\frac{\partial \vec{S}_{a}}{\partial t}\right\rangle_{S}=-\left\langle\vec{H} \times \vec{S}_{a}\right\rangle_{S}
$$

Eq.9 is none other than the equation of motion for precession of the spin $\vec{S}$ in the instantaneous field given by $\vec{H}$ of Eq. (47. We find that such classical equations of motion for a nonlocal in time action are exact in the quantum arena. [For affectionados of parafermion methods, we briefly note as an aside that although throughout we employed the bosonic spin coherent path integral representation, a similar result follows if the spinors $z$ were Grassmann variables (a net even number of permutations of the spinor coordinates are involved in proving Eq.(9).] The bulk of the paper will be devoted to a solution of Eq.97 for different realizations of a Josephson junction system.

We will momentarily dispense with the Keldysh contour indices. Due to the commutation relations $\vec{S} \times \vec{S}=i \vec{S}$, although the field $\vec{H}$ contains a piece which is linear in $\vec{S}$, the planar components of Eq. 9, may be reduced for certain problems to a linear equation in planar spin components $\left\langle S_{i}\right\rangle(i=x, y)$ which then must have the solution

$$
\left\langle S_{i}(t)\right\rangle=U_{i j}(t)\left\langle S_{j}(0)\right\rangle .
$$


We now invoke symmetry constraints. An external magnetic field $\vec{h}$ in the action (Eq. (2) lifts the $S U(2)$ spin rotational symmetry of the free spin leading in turn to a lower $U(1)$ symmetry of rotations about the external magnetic field axis. Such a symmetry is trivially encapsulated by the operator $R^{z}(\theta)$ rotating $\langle\vec{S}\rangle$ by an angle $\theta$ about the $\mathrm{z}$ (or magnetic field) axis. As a consequence, the evolution operator $U(t)$ of Eq. 10. must commute with $R^{z}(\theta)$. This, in turn, dictates that if the solution is in the form of Eq. 10, then the time evolution operator $U(t)$ must have the form

$$
U(t)=\left(\begin{array}{cc}
p(t) & q(t) \\
-q(t) & p(t)
\end{array}\right)
$$

Similarly, due the azimuthal rotational symmetry encapsulated by $R^{z}(\theta)$, the expectation value $\left\langle S_{z}(t)\right\rangle$ must be independent of $\left\langle S_{x}(0)\right\rangle$ and $\left\langle S_{y}(0)\right\rangle$. This form will indeed be borne out for our full Keldysh problem.

\section{THE KELDYSH BASIS EQUATIONS OF MOTION}

Within the non-equilibrium Keldysh formalism it is often advantageous to apply a simple linear transformation from the basis of up and down contour fields to the symmetric and antisymmetric linear combination of these fields. E.g., for the spin

$$
\begin{gathered}
\vec{S}_{c l} \equiv \frac{1}{2}\left(\vec{S}_{u p}+\vec{S}_{\text {down }}\right), \\
\vec{S}_{q u} \equiv\left(\vec{S}_{\text {up }}-\vec{S}_{\text {down }}\right) .
\end{gathered}
$$

The utility of this basis has its roots in the natural form for the various correlation functions- all simply related to the advanced, retarded, and "Keldysh" correlators. The subscripts "cl" and "qu" of Eq. 12 coding for "classical" and "quantum" suggest an intimate relation to classical and quantum Langevin like dynamics. We refer the uninitiated reader to excellent texts such as [20], [21] where the origin of this link is explored in depth. In Eq.12 we trivially generalize this change of basis to quantum spin systems. In this basis, when taken as operators in Eq. 12 [prior to a passage to a path integral representation], the spins no longer obey canonical commutation relations the spins no longer obey canonical commutations relations (e.g., $\left[\vec{S}_{q u}, \vec{S}_{c l}\right] \neq 0$ ) and are no longer normalized $\left(\vec{S}_{u p} \pm \vec{S}_{\text {down }}\right.$ may correspond to a spin-triplet, $S=1$, or to a spin singlet, $S=0$ ). Thus, we may not directly employ the $C P_{1}$ representation in this basis. For the current purposes, the equations of motion in this basis may be derived from Eq. (9) for the up and down contour spins,

$$
\begin{array}{r}
0=\left\langle\frac{d}{d t} S_{c l}^{k}+\left(\vec{h} \times \vec{S}_{c l}\right)_{k}+\int d t_{2} \epsilon_{i j k}\left(S_{c l}^{j}(t) S_{q u}^{j}(t)\right)\right. \\
\left(\begin{array}{cc}
\frac{K_{u u}+K_{u d}-K_{d u}-K_{d d}}{2} & \frac{K_{u u}-K_{u d}-K_{d u}+K_{d d}}{4} \\
\frac{K_{u u}+K_{u d}+K_{d u}+K_{d d}}{4} & \frac{K_{u u}-K_{u d}+K_{d u}-K_{d d}}{8}
\end{array}\right) \\
\left.\left(\begin{array}{c}
S_{c l}^{i}\left(t_{2}\right) \\
S_{q u}^{i}\left(t_{2}\right)
\end{array}\right)\right\rangle_{S},
\end{array}
$$

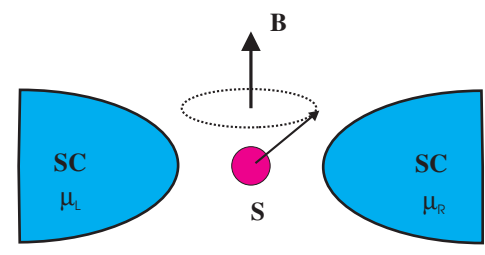

FIG. 2: Magnetic spin coupled to two superconducting leads.

and

$$
\begin{array}{r}
0=\left\langle\frac{d}{d t} S_{q u}^{k}+\left(\vec{h} \times \vec{S}_{q u}\right)_{k}+\int d t_{2} \epsilon_{i j k}\left(S_{c l}^{j}(t) S_{q u}^{j}(t)\right)\right. \\
\left(\begin{array}{cc}
K_{u u}+K_{u d}+K_{d u}+K_{d d} & \frac{K_{u u}-K_{u d}+K_{d u}-K_{d d}}{K_{u u}+K_{u d}-K_{d u}-K_{d d}} \\
2 & \frac{K_{u u}-K_{u d}-K_{d u}+K_{d d}}{4}
\end{array}\right) \\
\left.\left(\begin{array}{c}
S_{c l}^{i}\left(t_{2}\right) \\
S_{q u}^{i}\left(t_{2}\right)
\end{array}\right)\right\rangle_{S} .
\end{array}
$$

An average over $\exp [i S]$ is implicit in \langle\rangle$_{S}$. As emphasized earlier, these are not merely saddle point equations but are rather exact. In the above, although the time arguments were not explicitly written down, $K_{\alpha \beta}$ serves as a shorthand for $K_{\alpha \beta}\left(t, t_{2}\right)$.

\section{SINGLE SPIN DYNAMICS IN A JOSEPHSON JUNCTION}

\section{A. The system}

Our system is sketched in Fig. It consists of two identical ideal superconducting leads coupled each to a single spin; the entire system is further subjected to a weak external magnetic field. In Fig.(2), $\mu_{L, R}$ denote the chemical potentials of the left and right leads, $\vec{B}$ is a weak external magnetic field along the z-axis, and $\vec{S}=\left(S_{x}, S_{y}, S_{z}\right)$ is the operator of the localized spin. The wave-functions of our system are superpositions of the direct product of states of the left contact, the impurity spin, and the right contact,

$$
|\psi\rangle=\sum f_{L S R}\left(\left|\psi_{L}\right\rangle \otimes\left|\psi_{S}\right\rangle \otimes\left|\psi_{R}\right\rangle\right) .
$$

A tunneling matrix couples these different states. The Hamiltonian of this system reads

$$
\begin{array}{r}
\mathcal{H}=\mathcal{H}_{0}+\mathcal{H}_{T}, \\
\mathcal{H}_{0}=\mathcal{H}_{L}+\mathcal{H}_{R}-\mu B_{z} S_{z}, \\
\mathcal{H}_{T}=\sum_{\vec{k}, \vec{p}, \alpha, \alpha^{\prime}} e^{i \phi / 2} c_{R \vec{k} \alpha}^{\dagger}\left[T_{0} \delta_{\alpha \alpha^{\prime}}+T_{1} \vec{\sigma}_{\alpha \alpha^{\prime}} \cdot \vec{S}\right] c_{L \vec{p} \alpha^{\prime}}
\end{array}
$$

Here, $\mathcal{H}_{L}$ and $\mathcal{H}_{R}$ are the Hamiltonians in the left and right superconducting leads, while $c_{i k \alpha}^{\dagger}\left(c_{i k \alpha}\right)$ creates (annihilates) an electron in the lead a in the state $\vec{k}$ with spin $\alpha$ in the right/left lead for $i=L / R$ respectively. $\mathcal{H}_{L(R)}=\sum_{k(p) ; \sigma} \epsilon_{k(p)} c_{k(p), \sigma}^{\dagger} c_{k(p), \sigma}+$ 
$\frac{1}{2} \sum_{k(p) ; \sigma, \sigma^{\prime}}\left[\Delta_{\sigma \sigma^{\prime}}(k(p)) c_{k(p), \sigma^{\dagger}}^{\dagger} c_{-k(-p), \sigma^{\prime}}^{\dagger}+\right.$ h.c. $]$, where we denote the electron creation (annihilation) operators in the left (L) lead by $c_{k \sigma}^{\dagger}\left(c_{k \sigma}\right)$ while those in the right (R) lead by $c_{p \sigma}^{\dagger}$ $\left(c_{p \sigma}\right)$. The quantities $k(p)$ are momenta, $\sigma$ the spin index, while $\epsilon_{k(p), \sigma}$ and $\Delta_{\sigma \sigma^{\prime}}(k(p))$ are, respectively, the single particle energies of conduction electrons, and the pair potential in the leads. In Eq.16, the components $\vec{\sigma}_{\alpha \alpha^{\prime}}$ are entries of the three Pauli matrices $\left(\sigma_{\alpha \alpha^{\prime}}^{x}, \sigma_{\alpha \alpha^{\prime}}^{y}, \sigma_{\alpha \alpha^{\prime}}^{z}\right)$. In the current publication we consider s-wave symmetry pairing in the superconducting leads.Here, $\mu$ is the magnetic moment of the spin. With the spin embedded in the tunneling barrier, the conduction electron tunneling matrix becomes, spin-dependent [6] $\hat{T}=\left[T_{0} \hat{1}+T_{1} \vec{S} \cdot \vec{\sigma}_{c}\right]$. Here $T_{0}$ is a spin-independent tunneling matrix element and $T_{1}$ is a spin-dependent matrix element originating from the direct exchange coupling $J$ of the conduction electron spin $\vec{\sigma}_{c}$ to the localized spin $\vec{S}$. Henceforth, we will omit the $c$ subscript. We take both tunneling matrix elements ( $T_{0}$ and $\left.T_{1}\right)$ to be momentum independent. This is not a crucial assumption and is merely introduced to simplify notations. Typically, from the expansion of the work function for tunneling, $\frac{T_{1}}{T_{0}} \sim J / U$, where $U$ is the height of a spinindependent tunneling barrier [22]. A weak external magnetic field $B_{z} \sim 100$ Gauss will not influence the superconductors and we may ignore its effect on the leads. The operator $e^{i \phi / 2}$ is the (single electron) number operator. When the junction is linked to an external environment, the coupling between the junction and the environment induces fluctuations of the superconducting phase difference across the junction $(\phi(t))$.

\section{B. Physical Time Scales}

The Josephson junction with the spin has two time scales: (i) The Larmor precession frequency of the spin $\omega_{L}=$ $g \mu_{B} B \equiv h$, where $g, \mu_{B}$ are the gyromagnetic ratio and Bohr magneton of the conduction electron, respectively. (ii) The frequency $\omega_{J}=2 \mathrm{eV}$, with $e$ the electronic charge, characterizes the Josephson effect when an external voltage $V$ is applied across the junction.

\section{The Effective Action}

Josephson junctions are necessarily embedded into external electrical circuits. This mandates that the dynamics explicitly depends on the superconducting phase difference $\phi(t)$ across the junction. The evolution operator is given by the real-time path integral

$$
Z=\int D \phi D \vec{S} \exp [i S]
$$

The net action of Eq. (17) is given by $S=\left[\mathcal{S}_{\text {circuit }}(\phi)+\right.$ $\left.\mathcal{S}_{\text {spin }}(\vec{S})+\mathcal{S}_{\text {tunnel }}(\phi, \overrightarrow{\mathcal{S}})\right]$. The effective action $\mathcal{S}_{\text {tunnel }}$ contribution describes the junction itself. If all external fields are the same on both forward and backward branches of the Keldysh contour $(K)$ then $\mathcal{Z}=\operatorname{Tr} T_{K} \exp \left[-i \oint_{K} d t H_{T}(t)\right]=1$, where the trace is over both the electron and the spin degrees of freedom and $T_{K}$ denotes time ordering along the Keldysh contour. The label $\oint_{K}$ denotes integration along the Keldysh contour as shown in Fig. (11). We first take a partial trace in $\mathcal{Z}$ over the lead fermions (the bath) to obtain an effective spin action. The Josephson contribution to the resulting spin action reads $-\frac{1}{2} \oint_{K} d t \oint_{K} d t^{\prime}\left\langle T_{K} \mathcal{H}_{T}(\mathbf{S}(t), t) \mathcal{H}_{T}\left(\mathbf{S}\left(t^{\prime}\right), t^{\prime}\right)\right\rangle$, much in the spirit of Refs. [23, 24, 25]. For brevity, we set $A_{\sigma, \sigma^{\prime}} \equiv \sum_{k, p} c_{k \sigma}^{\dagger} c_{p \sigma^{\prime}}$. The tunneling Hamiltonian of a phase (voltage) biased junction

$$
\begin{array}{r}
\mathcal{H}_{T}=\left[T_{0} \delta_{\sigma \sigma^{\prime}}+T_{1} \mathbf{S} \cdot \sigma_{\sigma \sigma^{\prime}}\right] \\
\times\left(A_{\sigma \sigma^{\prime}} \exp (i \phi / 2)+A_{\sigma \sigma^{\prime}}^{\dagger} \exp (-i \phi / 2)\right) .
\end{array}
$$

In the presence of a dc voltage bias, $\phi=2 \mathrm{eVt}$. If $\phi$ is treated classically (i.e. $\phi$ is the same on the upper and the lower branches of the Keldysh contour), the contribution $\propto T_{0}^{2}$ to $\delta \mathcal{S}$ vanishes. The mixed contribution $\propto T_{0} T_{1}$ vanishes due to the singlet spin structure of the s-wave superconductor. The only surviving contribution reads

$$
\begin{gathered}
-\frac{T_{1}^{2}}{2} \oint_{K} d t \oint_{K} d t^{\prime}\left[\mathbf{S}(t) \cdot \sigma_{\alpha \beta}\right]\left[\mathbf{S}\left(t^{\prime}\right) \cdot \sigma_{\delta \gamma}\right] \\
\times\left(\left\langle T_{K} A_{\alpha \beta}(t) A_{\delta \gamma}\left(t^{\prime}\right)\right\rangle e^{i \frac{\phi(t)+\phi\left(t^{\prime}\right)}{2}}+\left(A, \phi \rightarrow A^{\dagger},-\phi\right)\right)(19)
\end{gathered}
$$

where we keep only the Josephson (off-diagonal) terms. The spin structure simplifies for the s-wave case:

$$
T_{1}^{2} \oint_{K} d t \oint_{K} d t^{\prime}\left[\mathbf{S}(t) \cdot \mathbf{S}\left(t^{\prime}\right)\right]\left[i D\left(t, t^{\prime}\right)\right]
$$

where the kernel $i D\left(t, t^{\prime}\right)$ is dictated by $\left\langle T_{K} A_{\uparrow \uparrow}(t) A_{\downarrow \downarrow}\left(t^{\prime}\right)\right\rangle e^{i \frac{\phi(t)+\phi\left(t^{\prime}\right)}{2}}+\left(A, \phi \rightarrow A^{\dagger},-\phi\right)$. The operators $A$ are bilinears in Fermi operators and thus the correlator $\left\langle T_{K} A_{\uparrow \uparrow}(t) A_{\downarrow \downarrow}\left(t^{\prime}\right)\right\rangle$ will amount to a sum of a product of two terms: a product of two normal Green's function $G$ and a product of two pair correlators $F$. Thus, generalizing the known effective tunneling action for a spin-less junction [23, 24, 25] to the new spin-dependent arena, we obtain

$$
\begin{array}{r}
\mathcal{S}_{\text {tunnel }}=-2 \oint_{K} d t \oint_{K} d t^{\prime} \alpha\left(t, t^{\prime}\right)\left[T_{0}^{2}+T_{1}^{2} \mathbf{S}(t) \cdot \vec{S}\left(t^{\prime}\right)\right] \\
\cos \left[\frac{\phi(t)-\phi\left(t^{\prime}\right)}{2}\right] \\
-2 \oint_{K} d t \oint_{K} d t^{\prime} \beta\left(t, t^{\prime}\right)\left[T_{0}^{2}-T_{1}^{2} \vec{S}(t) \cdot \vec{S}\left(t^{\prime}\right)\right] \\
\cos \left[\frac{\phi(t)+\phi\left(t^{\prime}\right)}{2}\right]
\end{array}
$$

where $i \alpha\left(t, t^{\prime}\right) \equiv G\left(t, t^{\prime}\right) G\left(t^{\prime}, t\right)$ and $i \beta\left(t, t^{\prime}\right) \equiv$ $F\left(t, t^{\prime}\right) F^{\dagger}\left(t, t^{\prime}\right)$. Here, the Green functions

$$
\begin{aligned}
G\left(t, t^{\prime}\right) & \equiv-i \sum_{\mathbf{k}}\left\langle T_{K} c_{\mathbf{k} \sigma}(t) c_{\mathbf{k} \sigma}^{\dagger}\left(t^{\prime}\right)\right\rangle, \\
F\left(t, t^{\prime}\right) & \equiv-i \sum_{\mathbf{k}}\left\langle T_{K} c_{\mathbf{k} \uparrow}(t) c_{-\mathbf{k} \downarrow}\left(t^{\prime}\right)\right\rangle, \\
F^{\dagger}\left(t, t^{\prime}\right) & \equiv-i \sum_{\mathbf{k}}\left\langle T_{K} c_{\mathbf{k} \uparrow}^{\dagger}(t) c_{-\mathbf{k} \downarrow}^{\dagger}\left(t^{\prime}\right)\right\rangle .
\end{aligned}
$$


We now express the spin action on Keldysh contour in the basis of coherent states

$$
\mathcal{S}_{\text {spin }}=-\oint_{K} d t \vec{h} \cdot \vec{S}+\mathcal{S}_{W Z N W}
$$

The second, Wess-Zumino-Novikov-Witten (WZNW), term in Eq. 25 depicts the Berry phase accumulated by the spin which we discussed earlier in the coherent spin representation wherein it amounts to a kinetic bilinear- the first term of Eq.(2)). In the calculations that follow we replace the spin measure $D S$ by the coherent spin state measure $D z D z^{*}$ and rely on our derived exact equations of motion. We now perform the Keldysh rotation of Eq. 12, defining the values of the spin and the phase variables. For the superconducting phase, we introduce (with notations following Refs. [23, 25])

$$
\phi \equiv \frac{1}{2}\left(\phi_{u p}+\phi_{\text {down }}\right), \chi \equiv \phi_{u p}-\phi_{\text {down }} .
$$

Within the Keldysh framework, the Josephson current is given by

$$
\langle I(t)\rangle=\frac{2 \pi}{\Phi_{0}}\left\langle\frac{\delta S}{\delta \chi(t)}\right\rangle,
$$

with $\Phi_{0}$ the unit fluxon (with full units restored, $\Phi_{0}=h c / e$ with $c$ the speed of light). With these definitions in hand, the tunneling part of the action reads

$$
\mathcal{S}_{\text {tunnel }}=\mathcal{S}_{\alpha}+\mathcal{S}_{\beta}
$$

where the normal (quasi-particle) tunneling part $\mathcal{S}_{\alpha}$ is expressed via the Green functions $\alpha^{R} \equiv \theta\left(t-t^{\prime}\right)\left(\alpha^{>}-\alpha^{<}\right)$and $\alpha^{K}(\omega) \equiv \alpha^{>}+\alpha^{<}$, where $i \alpha^{>}\left(t, t^{\prime}\right) \equiv G^{>}\left(t, t^{\prime}\right) G^{<}\left(t^{\prime}, t\right)$ and $i \alpha^{<}\left(t, t^{\prime}\right) \equiv G^{<}\left(t, t^{\prime}\right) G^{>}\left(t^{\prime}, t\right)$. Similarly the Josephsontunneling part $\mathcal{S}_{\beta}$ is expressed via the off-diagonal Green's functions $\beta^{R} \equiv \theta\left(t-t^{\prime}\right)\left(\beta^{>}-\beta^{<}\right)$and $\beta^{K}(\omega) \equiv \beta^{>}+\beta^{<}$, where $i \beta^{>}\left(t, t^{\prime}\right) \equiv F^{>}\left(t, t^{\prime}\right) F^{\dagger>}\left(t, t^{\prime}\right)$ and $i \beta^{<}\left(t, t^{\prime}\right) \equiv$ $F^{<}\left(t, t^{\prime}\right) F^{\dagger<}\left(t, t^{\prime}\right)$. The pair correlators $F^{<}\left(t, t^{\prime}\right)$ are derived from $F^{>}\left(t, t^{\prime}\right)$ by the interchange of $t$ with $t^{\prime}$. In the current article, we focus on the interaction between the supercurrent and the spin.

In Eq. 21], the normal-tunneling part $\mathcal{S}_{\alpha}$ is obtained from $\mathcal{S}_{\beta}$ by the following substitution: $\beta^{R / K}\left(t, t^{\prime}\right) \rightarrow \alpha^{R / K}\left(t, t^{\prime}\right)$, $\phi\left(t^{\prime}\right) \rightarrow-\phi\left(t^{\prime}\right)$, and $\chi\left(t^{\prime}\right) \rightarrow-\chi\left(t^{\prime}\right)$. The Keldysh terms (those including $\beta^{K}$ and $\alpha^{K}$ ), which normally give rise to random Langevin terms (see, e.g., Ref. [25]) are, in our case, suppressed at temperatures much lower than the superconducting gap $(T \ll \Delta)$, due to the exponential suppression of the correlators $\beta^{K}(\omega)$ and $\alpha^{K}(\omega)$ at $\omega<\Delta$.

To obtain $\beta^{R}$ we start from the Gor'kov Green functions

$$
\begin{aligned}
F^{>}\left(t, t^{\prime}\right) & =-i \sum_{k} \frac{\Delta}{2 E_{k}} e^{-i E_{k}\left(t-t^{\prime}\right)} \\
F^{>\dagger}\left(t, t^{\prime}\right) & =i \sum_{k} \frac{\Delta}{2 E_{k}} e^{-i E_{k}\left(t-t^{\prime}\right)}
\end{aligned}
$$

where the quasi-particle energy $E_{k} \equiv \sqrt{\Delta^{2}+\epsilon_{k}^{2}}$, with $\epsilon_{k}$ the free-conduction-electron dispersion in the leads. Putting all of the pieces together, we find that

$$
\beta^{R}\left(t-t^{\prime}\right)=-\theta\left(t-t^{\prime}\right) \sum_{k, p} \frac{\Delta^{2}}{2 E_{k} E_{p}} \sin \left[\left(E_{k}+E_{p}\right)\left(t-t^{\prime}\right)\right]
$$

The kernel $\beta^{R}\left(t-t^{\prime}\right)$ decays on (short) time scales of order $\mathcal{O}(\hbar / \Delta)$. Similarly,

$$
\beta^{K}\left(t-t^{\prime}\right)=-i \sum_{k, p} \frac{\Delta^{2}}{2 E_{k} E_{p}} \cos \left[\left(E_{k}+E_{p}\right)\left(t-t^{\prime}\right)\right]
$$

Henceforth, we will often employ the shorthand $\beta^{R / K}\left(t, t^{\prime}\right) \equiv \beta^{R / K}\left(t-t^{\prime}\right)$. Looking at Eq. 31, we see that the Fourier transform $\beta^{K}(\omega)$ vanishes for frequencies $\omega<\Delta$. This is not so for the retarded correlator $\beta^{R}$ due to the presence of the theta function. For now, we ignore the fluctuations in the superconducting phase and set $\phi_{\text {up }}(t)=\phi_{\text {down }}(t)=\phi(t)=\omega_{J} t$ with $\omega_{J}=2 \mathrm{eV}$ (and thus $\chi=0$ ). In this, "classical", limit

$$
\begin{aligned}
S_{\text {tunnel }} & \simeq 4 \int d t \int d t^{\prime} \beta^{R}\left(t, t^{\prime}\right) T_{1}^{2} \vec{S}_{q u}(t) \cdot \vec{S}_{c l}\left(t^{\prime}\right) j\left(t, t^{\prime}\right) \\
& +\int d t \int d t^{\prime} T_{1}^{2} \beta^{K}\left(t, t^{\prime}\right) \vec{S}_{q u}(t) \cdot \vec{S}_{q u}\left(t^{\prime}\right) j\left(t, t^{\prime}\right)(32)
\end{aligned}
$$

with $j\left(t, t^{\prime}\right) \equiv \cos \frac{\phi(t)+\phi\left(t^{\prime}\right)}{2}$.

\section{The Equations of Motion}

With the action at our disposal, we now write down the exact equations of motions and give a solution, exact to order $\mathcal{O}\left(T_{1}^{2}\right)$. Extracting, in the up-down contour basis, the coefficients, $\bar{K}_{a b}\left(t, t^{\prime}\right)$ of the $\vec{S}_{a}(t) \cdot \vec{S}_{b}\left(t^{\prime}\right)$ terms in Eq. (32), constructing $K_{a b}\left(t, t^{\prime}\right)$ from Eq. (4), and invoking Eq. 113, we find

$$
\begin{array}{r}
0=\left\langle\frac{d}{d t} \vec{S}_{c l}+\vec{h} \times \vec{S}_{c l}\right. \\
+4\left|T_{1}\right|^{2} \int d t^{\prime} j\left(t, t^{\prime}\right) \beta^{R}\left(t, t^{\prime}\right) \vec{S}_{c l}\left(t^{\prime}\right) \times \vec{S}_{c l}(t) \\
+2\left|T_{1}\right|^{2} \int d t^{\prime} j\left(t, t^{\prime}\right) \beta^{K}\left(t, t^{\prime}\right) \vec{S}_{q u}\left(t^{\prime}\right) \times \vec{S}_{c l}(t) \\
\left.+\left|T_{1}\right|^{2} \int d t^{\prime} j\left(t, t^{\prime}\right) \beta^{R}\left(t, t^{\prime}\right) \vec{S}_{q u}\left(t^{\prime}\right) \times \vec{S}_{q u}(t)\right\rangle_{S} \\
\equiv\left\langle\frac{d}{d t} \vec{S}_{c l}+\vec{h} \times \vec{S}_{c l}+\vec{I}_{c l-c l}+\vec{I}_{q u-c l}+\vec{I}_{q u-q u}\right\rangle_{S} .
\end{array}
$$

The final subscript $S$ serves to remind us that this is the path integral average computed with the action $S$. The various subscripts of the integrals $I$ denote the terms that they originate from (e.g. $I_{c l-c l}=4\left|T_{1}\right|^{2} \int d t^{\prime} j\left(t, t^{\prime}\right) \beta^{R}\left(t^{\prime}, t\right) \vec{S}_{c l}\left(t^{\prime}\right) \times$ $\vec{S}_{c l}(t)$ ). In Appendices B and C we outline, in detail, the evaluation of the various terms in Eq. 33. We will now solve Eq. 33 to order $\mathcal{O}\left(T_{1}^{2}\right)$. 


\section{E. Spin Dynamics in a Josephson Junction: An Exact Solution to $\mathcal{O}\left(T_{1}^{2}\right)$}

With all of the ingredients in place, we may now solve Eq. 33 to determine the spin dynamics to $\mathcal{O}\left(T_{1}^{2}\right)$. Henceforth, we will examine throughout the observable "classical" component of the spin $\vec{S}_{c l}$. To make the expressions more appealing we will dispense with the classical "cl" subscript. Similarly, the action $S$ subscript in all expectation values will be omitted as no time ordering subtleties appear below. We expand the spin as

$$
\langle\vec{S}(t)\rangle=\left\langle\vec{S}_{0}(t)\right\rangle+\langle\delta \vec{S}(t)\rangle
$$

Here, $\vec{S}_{0}$ is the solution to the (Larmor) problem of a single free spin in an external magnetic field. We computed the integrals borne by these zeroth order Larmor components in subsection XIC. Similarly, $\delta S(t)$ are the contributions borne by the retarded and Keldysh correlations. These corrections will lead to higher order contributions in $\langle\vec{I}\rangle$ which are irrelevant to our $\mathcal{O}\left(T_{1}^{2}\right)$ solution. We insert Eq. 34 into the equations of motion (Eqs. 33) and retain all terms to order $\mathcal{O}\left(\left|T_{1}\right|^{2}\right)$. This trivially leads to

$$
\begin{aligned}
\frac{d}{d t}\left\langle\delta S_{x}\right\rangle-\omega_{L}\left\langle\delta S_{y}\right\rangle+\left\langle I_{x}\right\rangle & =0 \\
\frac{d}{d t}\left\langle\delta S_{y}\right\rangle+\omega_{L}\left\langle\delta S_{x}\right\rangle+\left\langle I_{y}\right\rangle & =0 \\
\frac{d}{d t}\left\langle\delta S_{z}\right\rangle+\left\langle I_{z}\right\rangle & =0 .
\end{aligned}
$$

Here, $I_{\alpha=x, y, z}$ is the $\alpha$ direction component of $\left\langle\vec{I}_{c l-c l}+\right.$ $\left.\vec{I}_{q u-c l}\right\rangle$ which was computed in the previous subsection to or$\operatorname{der} \mathcal{O}\left(\left|T_{1}\right|^{2}\right)$. We see that the integrals $\vec{I}$ play the role of a driving force. Integrating, we find that

$$
\begin{aligned}
\left\langle\delta S_{z}(t)\right\rangle=\left|T_{1}\right|^{2}(1 & \left.-\cos \omega_{J} t\right)\left[\sum_{k, p} \frac{\Delta^{2} \omega_{L}}{E_{k} E_{p}\left(E_{k}+E_{p}\right)^{3}}\right. \\
& \left.+\left\langle S_{z}(0)\right\rangle \sum_{k, p} \frac{\Delta^{2}}{E_{k} E_{p}\left(E_{k}+E_{p}\right)^{2}}\right] .
\end{aligned}
$$

Differentiating the equation of motion for $\left\langle\delta S_{x, y}\right\rangle$ in Eq. 35 and inserting the equation of motion for $\left\langle\delta S_{y, x}\right\rangle$ we immediately obtain the equation of motion of a driven harmonic oscillator. A simple solution yields

$$
\begin{array}{r}
\left\langle\delta S_{x}(t)\right\rangle=c_{1} \cos \omega_{L} t+c_{2} \sin \omega_{L} t \\
+\sum_{\omega_{n}}\left(\frac{A_{n}}{\omega_{L}^{2}-\omega_{n}^{2}} \cos \omega_{n} t+\frac{B_{n}}{\omega_{L}^{2}-\omega_{n}^{2}} \sin \omega_{n} t\right)
\end{array}
$$

with

$$
\begin{aligned}
A_{\omega_{L}+\omega_{L J}} & =\left|T_{1}\right|^{2} \sum_{k, p} \frac{\Delta^{2}\left\langle S_{x}(0)\right\rangle\left(2 \omega_{L}^{2}+\omega_{J}^{2}+3 \omega_{L} \omega_{J}\right)}{2 E_{k} E_{p}\left(E_{k}+E_{p}\right)^{2}} \\
A_{\omega_{L}-\omega_{J}} & =\left|T_{1}\right|^{2} \sum_{k, p} \frac{\Delta^{2}\left\langle S_{x}(0)\right\rangle\left(2 \omega_{L}^{2}+\omega_{J}^{2}-3 \omega_{L} \omega_{J}\right)}{2 E_{k} E_{p}\left(E_{k}+E_{p}\right)^{2}} \\
B_{\omega_{L}+\omega_{J}} & =\left|T_{1}\right|^{2} \sum_{k, p} \frac{\Delta^{2}\left\langle S_{y}(0)\right\rangle\left(2 \omega_{L}^{2}+\omega_{J}^{2}+3 \omega_{L} \omega_{J}\right)}{2 E_{k} E_{p}\left(E_{k}+E_{p}\right)^{2}} \\
B_{\omega_{L}-\omega_{J}} & =\left|T_{1}\right|^{2} \sum_{k, p} \frac{\Delta^{2}\left\langle S_{y}(0)\right\rangle\left(2 \omega_{L}^{2}+\omega_{J}^{2}-3 \omega_{L} \omega_{J}\right)}{2 E_{k} E_{p}\left(E_{k}+E_{p}\right)^{2}}
\end{aligned}
$$

All in all, to $\mathcal{O}\left(T_{1}^{2}\right)$, the evolution of the planar spin components can be expressed in the format of Eqs. 1011 with

$$
\begin{aligned}
p(t) & =\cos \omega_{L} t+\left|T_{1}\right|^{2} \sum_{k, p} \frac{\Delta^{2}}{2 E_{k} E_{p}\left(E_{k}+E_{p}\right)^{2}} \\
& \times\left(\frac{\left(2 \omega_{L}^{2}+\omega_{J}^{2}+3 \omega_{L} \omega_{J}\right) \cos \left(\omega_{L}+\omega_{J}\right) t}{\omega_{L}^{2}-\left(\omega_{L}+\omega_{J}\right)^{2}}\right. \\
& \left.+\frac{\left(2 \omega_{L}^{2}+\omega_{J}^{2}-3 \omega_{L} \omega_{J}\right) \cos \left(\omega_{L}-\omega_{J}\right) t}{\omega_{L}^{2}-\left(\omega_{L}-\omega_{J}\right)^{2}}\right),
\end{aligned}
$$

and

$$
\begin{aligned}
q(t) & =\sin \omega_{L} t+\left|T_{1}\right|^{2} \sum_{k, p} \frac{\Delta^{2}}{2 E_{k} E_{p}\left(E_{k}+E_{p}\right)^{2}} \\
& \times\left(\frac{\left(2 \omega_{L}^{2}+\omega_{J}^{2}+3 \omega_{L} \omega_{J}\right) \sin \left(\omega_{L}+\omega_{J}\right) t}{\omega_{L}^{2}-\left(\omega_{L}+\omega_{J}\right)^{2}}\right. \\
& \left.+\frac{\left(2 \omega_{L}^{2}+\omega_{J}^{2}-3 \omega_{L} \omega_{J}\right) \sin \left(\omega_{L}-\omega_{J}\right) t}{\omega_{L}^{2}-\left(\omega_{L}-\omega_{J}\right)^{2}}\right) .
\end{aligned}
$$

This concludes our solution for the dynamics of a spin in a Josephson junction. Our analysis throughout centered on Josephson junctions composed of s-wave superconductors (see our starting point Eq.(20)). Slightly different quantitative results appear for other pairing symmetries (allowing, in theory, a determination of the pairing symmetry from observations of the spin/spin-wave dynamics and associated effects). The deviations from simple Larmor precessions are far stronger for triplet (i.e. odd angular momenta) superconductors.

\section{F. Physical Consequences: Josephson Nutations and Other Dynamical Effects}

We now discuss the physics behind our exact (to $\mathcal{O}\left(T_{1}^{2}\right)$ ) solution. Our solution provides testimony (and to new quantitative predictions) for several, inter-related, intriguing dynamical effects. We outline these below.

- Josephson Nutations:

In any system harboring a continuous rotational $U(1)$ symmetry about a certain axis (z), the orbital angular momentum $L_{z}$ is a constant of motion. Needless to say, the same trivially holds true for any spin system in which $\left[H, S_{z}\right]=0$ with 
$H$ the system Hamiltonian. In the presence of an external magnetic field along (or defining) the z-axis, as in the Larmor problem, the Hamiltonian $H=-h S_{z}$ commutes with $S_{z}$ and the longitudinal magnetization $\left\langle S_{z}(t)\right\rangle$ is a constant of motion. In our system with non local in time interactions triggered by superconducting pair correlations, such a conservation law no longer holds. Perusing Eq. 36, we find that the spin nutates above its average value. This occurrence for the $S=1 / 2$ is similar to that reported in [8] for macroscopic spin clusters $S \gg 1$. Here, however, the quantum fluctuations are profound for the $S=1 / 2$ case and lead to strong deformations of the nutation shape. The physical engine behind the nutations is the small time separation between the two tunneling electrons forming the Cooper pair. As the "first" electron tunnels through, it exerts a torque on the spin. A certain time later (of order $\hbar / \Delta$ with dimensions restored) after the spin $\vec{S}$ has already revolved a small amount, the opposite spin member of the Cooper pair tunnels through and exerts a torque of an opposite sign on the spin $\vec{S}$. Due to the small time lag between the two tunneling electrons, the two opposite sign torques exerted on $\vec{S}$ by the two opposite sign spins of the tunneling singlet do not cancel and lead to a net effect. This origin is made evident in the retarded correlations $\beta^{R}$ which further spark a non-vanishing driving force $\left\langle I_{c l-c l}\right\rangle$ along the z-axis. Mathematically, all of this results as the tunneling portion of the action contains terms which trivially do not conserve $S_{z}$. In the aftermath, this led to an effective time dependent force acting on $S_{z}$. Its form may be seen by examining the integral $\left\langle I_{z}\right\rangle$ appearing in Eq. 35. The latter is the z-component of the integrals $\left\langle\vec{I}_{c l-c l}\right\rangle$ and $\left\langle\vec{I}_{q u-c l}\right\rangle$ appearing in Eqs. 66 67. (Needless to say, if both members of the Cooper pair share the same polarization (as in triplet superconductors) then a far greater effect results.)

A manifestation of the resulting dynamical effect as a consequence of these effective external forces in conventional (swave) Josephson Junctions is vividly seen in Eq. (36. We have derived similar expressions via an independent density matrix approach [26]. An exaggerated schematic of this effect is provided in Fig. (3) which, qualitatively, is none other than the standard illustration for classical rigid body nutations. We find that such motions now appear in the quantum arena for a single $S=1 / 2$ particle! The precise shape of our trajectories, however, is markedly different from that exhibited by classical rigid rotors.

- Spin Contractions and Effective Longitudinal Fields:

Glancing at Eq.67, the reader will see that the effective $\left\langle\vec{I}_{q u-c l}\right\rangle$ can be seen to dilate the spin (the uniform contribution proportional to $\langle\vec{S}(t)\rangle$ in the second equality of Eq.67) and in unison to effectively emulate a time dependent magnetic field $\overrightarrow{\delta h}_{e f f} \propto \hat{e}_{z} \cos \phi(t)$ along the z-axis in the spin equation of motion, $d\langle\vec{S}\rangle / d t=\ldots+\langle\vec{S}\rangle \times \overrightarrow{\delta h}_{e f f}$. Both of these effects were noted in [9]. In Eq. 67, we explicitly see their origin. The uniform contraction is triggered by an entanglement of the tunneling electrons with our $S=1 / 2$ particle. We now very briefly elaborate on the physics of this statement for the benefit of general readers. The expectation values $\langle\vec{S}\rangle$ amount to weighted sums over all possible states $|\psi\rangle$

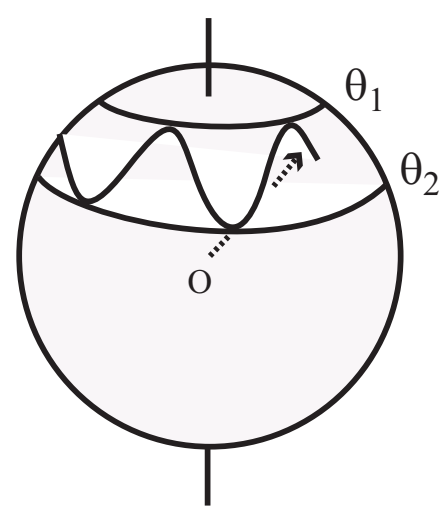

FIG. 3: The resulting spin motion on the unit sphere in the general case. As in the motion of classical spinning top, the spin exhibits undulations along the polar direction. As a consequence of entanglement with the tunneling electrons, the magnitude of the spin is not constant- the spin further "breaths" in and out as it nutates.

(see Eq.15). In any pure (i.e. unentangled) state of a spin$1 / 2$ problem, the sum $\left[\left\langle S_{x}\right\rangle^{2}+\left\langle S_{y}\right\rangle^{2}+\left\langle S_{z}\right\rangle^{2}\right]=1 / 4$ - the spin expectation values lie on the Bloch sphere. Entanglement in a spin-1/2 problem such as ours is marked by a contraction, $\left[\left\langle S_{x}\right\rangle^{2}+\left\langle S_{y}\right\rangle^{2}+\left\langle S_{z}\right\rangle^{2}\right]<S^{2}=1 / 4$. Any single spin expectation value within the Bloch sphere, $|\langle\psi|\vec{S}| \psi\rangle|<S$, denotes an expectation value computed in a multi-particle state $|\psi\rangle$ which is entangled. In the case here $|\psi\rangle$ spans the single spin and the tunneling electrons. Such a time dependent contraction in the norm of $\langle\vec{S}\rangle$ relative to the Bloch radius is evident in our exact solution of Eqs. 10,11, 39, 40, 34, 36.

\section{- Nonlinear planar precession:}

A notable facet of the dynamics given by the effects discussed above are non-uniform planar precessions. We find that within the plane transverse to the applied field direction, the azimuthal angle describing the spin orientation, $\varphi(t)=$ $\tan ^{-1}\left(\left\langle S_{y}(t)\right\rangle /\left\langle S_{x}(t)\right\rangle\right)$ is no longer a linear in time. This effect bears, once again, strong semblance to nutations in classical rigid body dynamics. In the Larmor problem of a free spin in a magnetic field, $\varphi(t)=\omega_{L} t$. In our case, the precession about the applied field direction is no longer uniform. Its form is encapsulated in Eqs. (37/38 or, alternatively, by Eqs. 10 113940. Once again, mathematically, the origins of this effect are rooted in the effective planar (xy) components of the effective force $\langle\vec{I}\rangle$ appearing in Eq. 35 . The explicit form of this effective force is given by the sum of the two integrals evaluated in Eqs. 66 67 and whose origin explicitly lies, once again, in the same non-local in time correlations borne by the superconducting correlations.

In summary, all of the above qualitative findings for the problem a single $S=1 / 2$ spin inserted in a Josephson junction are made vivid in our $\mathcal{O}\left(T_{1}^{2}\right)$ exact solution. From Eqs. 101139 40 for the planar spin components and from Eqs. 34 36 for the longitudinal spin we clearly see how all of these effects come into play. 


\section{RETARDED CORRELATIONS IN GENERAL SPIN $S$ DYNAMICS IN A JOSEPHSON JUNCTION}

The equation of motion, Eq. (9), is valid for all spins $S$. Much of our formalism follows with no change. We now examine the integrals $\langle\vec{I}\rangle$ in the general spin $S$ problem.

We find that for a spin of size $S$, the integral $\left\langle\vec{I}_{q u-c l}\right\rangle$ undergoes no change relative to its $S=1 / 2$ form- Eq. 67) remains the same. The associated physics fleshed out in the second equality of Eq.67) which was described in the previous section (spin contractions and the presence of an effective longitudinal field) undergoes no change for the general spin $S$ case.

Next, we evaluate $\left\langle\vec{I}_{c l-c l}\right\rangle$. For large spins, $S \gg 1$, the product $\left\langle\vec{S}(t) \times \vec{S}\left(t^{\prime}\right)\right\rangle$ is well approximated by the vector product of averages $\langle\vec{S}(t)\rangle \times\left\langle\vec{S}\left(t^{\prime}\right)\right\rangle$. Then, the approach of Ref. [8] is well justified and we can obtain the Josephson nutations of a big spin. For any $S$ and $t^{\prime}>t$ we obtain

$$
\begin{aligned}
& \left\langle\vec{S}_{c l}\left(t^{\prime}\right) \times \vec{S}_{c l}(t)\right\rangle_{S} \\
= & \frac{1}{2}\left\langle\left[\left\{S_{y}(t), S_{z}(t)\right\}_{+}\left[\cos \omega_{L}\left(t^{\prime}-t\right)-1\right]\right.\right. \\
- & \left.\left\{S_{x}(t), S_{z}(t)\right\}_{+} \sin \omega_{L}\left(t^{\prime}-t\right)\right] \hat{e}_{x} \\
+ & {\left[\left\{S_{x}(t), S_{z}(t)\right\}_{+}\left[1-\cos \omega_{L}\left(t^{\prime}-t\right)\right]\right.} \\
- & \left.\left\{S_{y}(t), S_{z}(t)\right\}_{+} \sin \omega_{L}\left(t^{\prime}-t\right)\right] \hat{e}_{y} \\
+ & \left.\left(2 S_{x}^{2}(t)+2 S_{y}^{2}(t)\right) \sin \omega_{L}\left(t^{\prime}-t\right) \hat{e}_{z}\right\rangle,
\end{aligned}
$$

where $\{\ldots\}_{+}$denotes an anticommutator. In the $S=1 / 2$ case, the integral stemming from these vectorial product correlations is parallel to the $\mathrm{z}$-axis, $\left\langle\vec{I}_{c l-c l}\right\rangle \| \mid \hat{e}_{z}$. For spins of size $S>1 / 2$, however, as we see from Eq. 411, the planar $(x, y)$ components also come to the fore and lead to retarding correlation $\left(\beta^{R}\right)$ effects in Eq. 33. Furthermore, the magnitude of the driving force $\left\langle I_{c l-c l ; z}\right\rangle$ along the $\mathrm{z}$-axis, much unlike the $S=1 / 2$ case is time dependent.

For general spins of size $S>1 / 2$, both retarded $\left(\beta^{R}\right)$ and Keldysh $\left(\beta^{K}\right)$ are non-zero along any spin direction. All of the effects discussed in subsection VF are present.

It is noteworthy to discuss the scaling of all terms with the spin size $S$. As evident from Eq.41, the integral $\left\langle\vec{I}_{c l-c l}\right\rangle$ spawned by retarded correlations scales as $S^{2}$. Similarly, as seen from Eq. 67, whose form holds for arbitrary $S$, the effective driving force $\left\langle\vec{I}_{q u-c l}\right\rangle$ generated by Keldysh correlations $\left(\beta^{K}\right)$ scales as $S$, i.e. $\left\langle\vec{I}_{q u-c l}\right\rangle \alpha S$. Thus, for large spins $S \gg 1$, the retarded contributions overwhelm stochastic Keldysh contributions. In the classical limit, $S \rightarrow \infty$, only the retarded contributions remain [8].

\section{SPIN TRIGGERED AC EFFECTS}

Thus far our discussion centered on a Josephson junction for a time independent potential difference $V$ (dc voltage bias) between the two superconducting leads for which $\phi(t)=\omega_{J} t$ with $\omega_{J}=2 \mathrm{eV}$.
We now briefly sketch matters for an ac voltage bias wherein the potential drop is oscillatory in time and the corresponding phase difference is $\phi(t)=A \sin \Omega t$. To make the physics more transparent, we omit any dc contributions to the voltage (and thus linear in time contributions to the phase). This serves as a caricature of rf driven Josephson junctions known to exhibit the famous Shapiro steps [27].

The setup is given by Fig. 2 for a spin $S=1 / 2$ particle yet now with an ac voltage applied across the junction. In the sections that follow, we will resume our central focus on the constant voltage drop case, $\phi(t)=\omega_{J} t$. Only in this short section do we analyze an applied ac voltage bias.

The calculations for the ac voltage bias case parallel the analysis of the previous sections. First, we express all terms by pure harmonics. This is readily achieved by relying on the identity

$$
e^{i C \sin x}=\sum_{n} J_{n}(C) e^{i n x},
$$

with $\left\{J_{n}(C)\right\}$ Bessel functions. The factor $j\left(t, t^{\prime}\right)$ of Eq. 32 and thereafter now becomes

$$
j\left(t, t^{\prime}\right)=\sum_{n, m} J_{n}\left(\frac{A}{2}\right) J_{m}\left(\frac{A}{2}\right) \cos \left[\Omega\left(n t+m t^{\prime}\right)\right] .
$$

The analog of Eq. 66 for the ac voltage bias case is

$$
\begin{aligned}
\left\langle\vec{I}_{c l-c l}\right\rangle_{S}=-\left|T_{1}\right|^{2} \hat{e}_{z} \sum_{k, p} & \frac{\Delta^{2}}{E_{k} E_{p}} \sum_{n, m} J_{n}\left(\frac{A}{2}\right) J_{m}\left(\frac{A}{2}\right) \\
& \times \frac{2 m \Omega \omega_{L} \sin \Omega(n+m) t}{\left(E_{k}+E_{p}\right)^{3}} .
\end{aligned}
$$

Further resonant (delta function) terms make an appearance for $m \ggg 1$.

Similarly, the analog of Eq.67) reads

$$
\begin{array}{r}
\left\langle\vec{I}_{q u-c l}\right\rangle_{S}=-\left|T_{1}\right|^{2} \sum_{n, m, k, p} \frac{J_{n}\left(\frac{A}{2}\right) J_{m}\left(\frac{A}{2}\right) \Delta^{2}}{E_{k} E_{p}\left(E_{k}+E_{p}\right)^{2}} \\
\times\left[\left(2 m \Omega\left\langle S_{x}(t)\right\rangle \sin (n+m) \Omega t-\left\langle S_{y}(t)\right\rangle \omega_{L} \cos (n+m) \Omega t\right) \hat{e}_{x}\right. \\
+\left(2 m \Omega\left\langle S_{y}(t)\right\rangle \sin (n+m) \Omega t+\left\langle S_{x}(t)\right\rangle \omega_{L} \cos (n+m) \Omega t\right) \hat{e}_{y} \\
\left.+2 m \Omega\left\langle S_{z}(0)\right\rangle \sin (n+m) \Omega t \hat{e}_{z}\right] .
\end{array}
$$

To $\mathcal{O}\left(\left|T_{1}\right|^{2}\right)$, the nutations are given by

$$
\begin{array}{r}
\left\langle\delta S_{z}(t)\right\rangle=\left|T_{1}\right|^{2} \sum_{k, p} \frac{\Delta^{2}}{E_{k} E_{p}} \sum_{n+m \neq 0} J_{n}\left(\frac{A}{2}\right) J_{m}\left(\frac{A}{2}\right) \\
\times \frac{2 m \Omega}{\left(E_{k}+E_{p}\right)^{2}}\left(\frac{\omega_{L}}{E_{k}+E_{p}}+\left\langle S_{z}(0)\right\rangle\right) \\
\times \frac{1-\cos \Omega(n+m) t}{n+m} .
\end{array}
$$

Higher order effects further enhance this response. Eq.45 is the ac voltage bias analog of Eq. (36) for the dc voltage bias case. The seminal feature of our results is the existence of frequencies in the spin dynamics of all integer multiples of the 
voltage bias driving ac frequency $\Omega$. As the spin alters (via back-action effects) the tunneling supercurrent, the supercurrent will exhibit oscillations at all frequencies $\omega_{r}=r \Omega$ with $r$ an integer. Extending the results of [9] to this problem, the supercurrent

$$
\begin{array}{r}
\langle I(t)\rangle=\sin \phi(t)\left[2 \pi^{2} e \rho^{2} \Delta\left(\left|T_{0}\right|^{2}-\frac{3}{4}\left|T_{1}\right|^{2}\right)\right. \\
\left.+4 e\left|T_{1}\right|^{2} \rho^{2} h\left\langle S_{z}(t)\right\rangle\right],
\end{array}
$$

where $\rho$ is the spin density of states within the leads, with the spin given by Eqs. 34 45 with the Larmor $\left\langle S_{z}(t)\right\rangle_{0}=$ $\left\langle S_{z}(0)\right\rangle$.

\section{FERROMAGNETS IN JOSEPHSON JUNCTIONS}

We now investigate what transpires when ferromagnets (instead of a single spin) are immersed between two s-wave superconductors with a dc bias voltage applied across the junction (as illustrated in Fig.(4)). As in the single spin problem, the full problem involves both the back-action of the spin on the phase of the superconductors (ignored here) and the spin dynamics sparked by the tunneling current (which we focus on below). Further, for extended junctions, phasons naturally appear. In what follows, we assume that the phases of the two superconductors surrounding a single magnetic slab have a spatially uniform phase difference $\phi(t)$. The tunneling action amounts to a sum over individual tunneling actions through each of the individual spins labeled by their sites $\vec{r}$,

$$
\begin{array}{r}
S_{\text {tunnel }} \simeq \\
4 \sum_{\vec{r}} \int d t \int d t^{\prime} \beta^{R}\left(t, t^{\prime}\right) T_{1}^{2} \vec{S}_{q u}(\vec{r}, t) \cdot \vec{S}_{c l}\left(\vec{r}, t^{\prime}\right) j\left(t, t^{\prime}\right) \\
+\int d t \int d t^{\prime} T_{1}^{2} \beta^{K}\left(t, t^{\prime}\right) \vec{S}_{q u}(\vec{r}, t) \cdot \vec{S}_{q u}\left(\vec{r}, t^{\prime}\right) j\left(t, t^{\prime}\right) .
\end{array}
$$

For ferromagnetic spin chains/planes with arbitrary exchange constants $J\left(\vec{r}, \vec{r}^{\prime}\right)$, and scaled external magnetic field $\vec{h}$, the exact equation of motion reads

$$
\begin{array}{r}
0=\left\langle\frac{d \vec{S}_{c l}(\vec{r})}{d t}+\vec{h} \times \vec{S}_{c l}(\vec{r})+\vec{I}_{c l-c l ; r}+\vec{I}_{q u-c l ; r}\right. \\
\left.+\sum_{\vec{r}^{\prime}} J\left(\vec{r}, \vec{r}^{\prime}\right) \vec{S}_{c l}\left(\vec{r}^{\prime}, t\right) \times \vec{S}_{c l}(\vec{r}, t)\right\rangle_{S} .
\end{array}
$$

It is hard not to notice a resemblance between the single spin problem (Eq. (33) and the problem of the ferromagnet (Eq.477). Indeed, as we will shortly demonstrate the spin wave dynamics in the ferromagnet within a Josephson junction bears much in common with the single spin problem with the proviso that the various ferromagnetic spin waves feel an effective momentum dependent magnetic field of strength $h_{\text {eff }}=h+S[J(\vec{k})-J(0)]$ with $J(\vec{k})$ the Fourier transform of the two spin interaction $J\left(\vec{r}, \vec{r}^{\prime}\right)$.

The solution proceeds much the same as for the single spin problem. Henceforth, we discuss the qualitative physics expected. Unlike the precise solutions presented till now, what

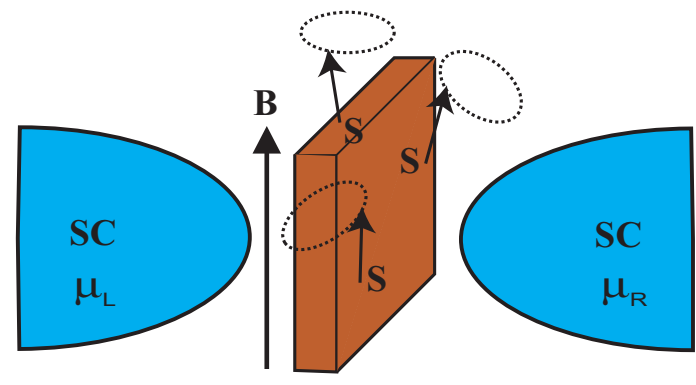

FIG. 4: A ferromagnetic slab inserted between two superconducting leads. The entire system is subjected to a weak external magnetic field $B$. A schematic of the precessing spins is shown.

follows is a quick qualitative sketch by way of an analogy. An exact solution will be detailed elsewhere [29].

Transforming from spin variables to bosonic operators $(b(\vec{r}))$ at all lattice sites $\vec{r},[28]$

$$
\begin{array}{r}
S^{+}(\vec{r})=b^{\dagger}(\vec{r}) \sqrt{2 S}, \\
S^{-}(\vec{r})=\left[b(\vec{r})-\frac{1}{2 S} b^{\dagger}(\vec{r}) b(\vec{r}) b(\vec{r})\right] \sqrt{2 S}, \\
S_{z}=-S+b^{\dagger}(\vec{r}) b(\vec{r}) .
\end{array}
$$

Sans the $\mathcal{O}\left(\left|T_{1}\right|^{2}\right)$ tunneling part of the action, the action is quadratic in the bosonic operators and is readily diagonalized in $\vec{q}$ space. We find that the free part of the action

$$
\begin{array}{r}
S_{0}=-\int d t \int \frac{d^{d} q}{(2 \pi)^{d}}\{S[J(\vec{q})- \\
\quad J(0)]+h\} \\
\times b^{*}(\vec{q}) b(\vec{q}),
\end{array}
$$

with $d$ the dimension of the inserted magnet. (As the problem is ferromagnetic, $\left.J(0)=\min _{\vec{q}}\{J(\vec{q})\}\right)$. Comparing this action to the one appearing in the single spin problem, we find that to Gaussian order the spin-wave problem is identical to the dynamics of a single spin with the replacement

$$
h \rightarrow h_{e f f}(\vec{q}) \equiv\{S[J(\vec{q})-J(0)]+h\} .
$$

The quadratic contribution of the $\mathcal{O}\left(\left|T_{1}\right|^{2}\right)$ portion of the action involving non-local in time correlations has precisely the same form for both the single spin problem and for each mode $\vec{q}$ of the spin-wave problem. Thus, the quadratic in $b, \mathcal{O}\left(\left|T_{1}\right|^{2}\right)$ corrections to the spin dynamics are given by Eqs. 3637 with the replacement of Eq. 50).

For instance, the above analogy suggests that that the net ferromagnetic moment variation in $S=1 / 2$ ferromagnets is

$$
\begin{array}{r}
\frac{\delta M}{V} \\
=\left|T_{1}\right|^{2}\left(1-\cos \omega_{J} t\right)\left[\sum_{k, p} \frac{\Delta^{2} \omega_{L}}{E_{k} E_{p}\left(E_{k}+E_{p}\right)^{3}}\right. \\
\left.+\frac{M}{V} \sum_{k, p} \frac{\Delta^{2}}{E_{k} E_{p}\left(E_{k}+E_{p}\right)^{2}}\right],
\end{array}
$$

with $V$ the volume of the magnet and $M$ its magnetization sans the supercurrent. Alternatively, the analysis may parallel 
the derivation of the previous sections word for word while taking the unperturbed solution (the analogue of the Larmor solution of Eq.61) to be a spin wave and computing all corrections to $\mathcal{O}\left(T_{1}^{2}\right)$.

In the continuum limit,

$$
h_{e f f}(\vec{q})=\frac{\rho_{s}}{m_{0}} q^{2}+h,
$$

with $m_{0} \equiv S / v$ (where $v$ is the volume per site), the magnetization density of the ground state, and $\rho_{s}$ the spin stiffness.

This $h \rightarrow h_{\text {eff }}(\vec{q})$ correspondence applies to any property inherited by the single spin dynamics in the Josephson junction. In particular, in [9] it was beautifully shown how spin dynamics may alter the super-current in the Junction. The current may be computed by the likes of Eq.27. Extending these results to a ferromagnet inserted in a Josephson junction by the correspondence of Eq. 50, we find that the new spin wave dynamics leads to the supercurrent,

$$
\begin{array}{r}
\langle I(t)\rangle=\sin \phi(t) \int \frac{d^{d} q}{(2 \pi)^{d}}\left[2 \pi^{2} e \rho^{2} \Delta\left(\left|T_{0}\right|^{2}-\frac{3}{4}\left|T_{1}\right|^{2}\right)\right. \\
\left.+4 e\left|T_{1}\right|^{2} \rho^{2} h_{e f f}(\vec{q})\left\langle S_{z}(\vec{q})\right\rangle\right]
\end{array}
$$

with $\rho$ the spin density of states within the leads. A matching of the Josephson and spin frequencies (such as present here for variations in the low temperature magnetic moment (see Eqs. 3651)) leads to a DC signal; additional harmonics further appear. We emphasize that in the above we compared only the Gaussian portion in the Bose fields. Higher order (non-Gaussian) terms originating from Eq. 48 as well as phasons alter the natural correspondence of Eq.(50). A full discussion of these issues will be detailed elsewhere [29].

\section{OTHER GEOMETRIES}

If phason contributions are neglected, then by a trivial change of geometry all of our results thus far, will apply for other systems as well. For instance, by replacing one of the superconducting leads by a surface, the resulting system may emulate a superconducting tip coupled to superconducting surface through a single spin or a ferromagnet. Here, all of the results of Sections $\nabla \nabla I I I$ for the spin dynamics and tunneling current hold.

Similarly, by replacing both superconducting leads by surfaces and examining a magnetic layer inserted in between, the resultant system looks much alike a layered superconducting/magnetic system. In this system, the results of Section VIII apply.

\section{LARGE $S$ ADIABATIC APPROXIMATIONS}

Thus far we studied the dynamics of single spins and of ferromagnets. In [8], the large $S$ limit of the single spin problem was studied. In that work, several approximations were made:

(i) The perturbative approach that we employed in the current article which allows an exact evaluation of all pertinent integrals to low orders was replaced by an "adiabatic" approximation wherein the slow dynamics of the spin vis a vis electronic processes was explicitly incorporated, $\vec{S}\left(t^{\prime}\right) \simeq$ $\vec{S}(t)+\left(t^{\prime}-t\right)(d \vec{S} / d t)$.

(ii) The ("classical") large $S$ limit allowed us to omit many instances of $\vec{S}_{q u}$ in the equations of motion and only $\beta^{R}$ related contributions in the tunneling action were consequential. Furthermore, as briefly alluded to earlier, in this limit, the average of the vectorial product $\left\langle\vec{S}\left(t^{\prime}\right) \times \vec{S}(t)\right\rangle$ is equal to the product of the averages $\left\langle\vec{S}\left(t^{\prime}\right)\right\rangle \times\langle\vec{S}(t)\rangle$. Correspondingly, any expectation value braces may be omitted. Thus, we may replace any expectation value $\langle\mathcal{A}\rangle$ by $\mathcal{A}$ itself.

The advantage of this method is that furnishes an elegant non-perturbative closed form solution for the spin dynamics. We will not repeat the results for the single spin cluster $(S \gg 1)$ problem here and rather refer the reader to [8]. We now briefly comment on applications of this method to other systems.

To $\mathcal{O}\left(\left|T_{1}\right|^{2}\right)$, the spin wave dynamics in ferromagnets may be attained via the substitution of Eq. 50. Equivalently, the spin wave equations of motion may also be determined directly when applying the adiabatic approximation on Eq.47. We then find

$$
\begin{array}{r}
\frac{d \vec{S}_{c l}\left(\vec{r}_{i}\right)}{d t}+\vec{h} \times \vec{S}_{c l}\left(\vec{r}_{i}\right)+\sum_{j} J_{i j} \vec{S}_{c l}\left(\vec{r}_{j}, t\right) \times \vec{S}_{c l}\left(\vec{r}_{i}, t\right) \\
+\kappa \vec{S}_{c l} \times \frac{d \vec{S}_{c l}}{d t} \sin \omega_{J} t=0(54)
\end{array}
$$

with $\kappa \equiv \sum_{k, p} \frac{|\Delta|^{2}\left|T_{1}\right|^{2}}{E_{k} E_{p}}\left[\left(E_{k}+E_{p}-e V\right)^{-2}-\left(E_{k}+E_{p}+\right.\right.$ $\left.e V)^{-2}\right]$. The appropriate spin wave equation is

$$
\begin{aligned}
\frac{d b(\vec{q})}{d t}=i[h+ & S\{J(\vec{q})-J(0)\}] b(\vec{q}) \\
& +\kappa \partial_{t} b(\vec{q}, t) \sin \omega_{J} t
\end{aligned}
$$

The solution to Eq.55 is

$$
\begin{array}{r}
b(\vec{q}, t)=b(\vec{q}, 0) \exp \left[-\frac{2 i(S\{J(\vec{q})-J(0)\}+h)}{\omega_{J} \sqrt{1-\kappa^{2}}}\right. \\
\left.\times\left\{\tan ^{-1}\left(\frac{\kappa}{\sqrt{1-\kappa^{2}}}\right)-\tan ^{-1}\left(\frac{\kappa-\tan \left(\omega_{J} t / 2\right)}{\sqrt{1-\kappa^{2}}}\right)\right\}\right],
\end{array}
$$

which is quite different from the standard spin-wave evolution in a magnet outside a Josephson junction. The key feature is a nonuniform evolution of each spin-wave. Similar to the azimuthal precession of a single spin, the planar components $S_{x, y}$ precess as the real and imaginary parts of $\exp [i \varphi(t)]$ with a nonlinear $\varphi(t)$. Thermodynamic quantities computed via the corrected bosonic spin-wave dispersion exhibit corrections.

Similarly, we may examine the adiabatic large $S$ equations of motion for an antiferromagnetic spin chain oriented along the $\mathrm{z}$-axis in a Josephson junction (just as in Fig.(4) yet now with a single antiferromagnetic spin chain replacing the ferromagnetic slab in an $h=0$ background). We then find that that the staggered spin, $\overrightarrow{\tilde{S}}_{i} \equiv(-1)^{i} \vec{S}_{i}$ (with the integer $i$ the spin 
site location along the chain) satisfies

$$
\begin{array}{r}
0=\square \overrightarrow{\tilde{S}}_{c l}(t)+3 \partial_{t} \overrightarrow{\tilde{S}}_{c l}(t) \times \partial_{z} \overrightarrow{\tilde{S}}_{c l}(t) \\
+\kappa \partial_{t} \overrightarrow{\tilde{S}}_{c l}(t) \sin \omega_{J} t
\end{array}
$$

where $\square \equiv \frac{v_{s}}{g}\left[\partial_{z}^{2}-\frac{1}{v_{s}^{2}} \partial_{t}^{2}\right]$. Here, $g=2 / S$ and the spin wave velocity $v_{s}=2 a J S$, with $a$ is the lattice constant. The role of the supercurrent as an effective driving term is evident in the last line of Eq.57.

\section{CONCLUSIONS}

In conclusion, our work addresses new dynamical effects exhibited by spins in Josephson Junctions. En route, many features (general and specific) were found:

(1) We derived the exact equation of motion for spin systems on Keldysh contours.

(2) The $S=1 / 2$ spin dynamics of a single spin in a Josephson junction was investigated and a perturbative solution was given. Spin-1/2 Josephson nutations are predicted.

(3) Spin dynamically triggered ac effects are predicted.

(4) The spin wave dynamics of a ferromagnet in between two superconducting leads was investigated. We predict nontrivial spin wave dynamics as well as new manifestations of this dynamics (most notably in the supercurrent).

(5) Large $S$ expressions were discussed for ferromagnetic slabs and antiferromagnetic spin chains in a Josephson junction.

\section{Acknowledgments}

This work was supported by the US DOE under LDRD X1WX (ZN and AVB). We gratefully acknowledge discussions with Yu Makhlin.

\section{A. Appendix A: Detection}

The non-trivial spin-wave and associated supercurrent in Josephson junctions containing ferromagnets (section VIII) may be seen more readily seen than those of single spins. The spin dynamics may be discerned by measuring the magnetization of the ferromagnetic slab as a function of time (as suggested by Eq. (51) as well as by monitoring the supercurrent (given by Eq.53). Other techniques may involve standard measurements of microwave radiation from the junction (and backaction effects). The magnitudes of these effects will be studied elsewhere [29].

We now briefly review a detection scheme discussed in [8], [30] for the Josephson nutations for the $S \gg 1$ limit of the general spin $\mathrm{S}$ results of Section(VI). This corresponds to a single magnetic cluster.

As it moves, the spin cluster magnetic moment generates a time-dependent magnetic field, $\delta \vec{B}(\mathbf{r}, t)=\frac{\mu_{0}}{4 \pi}[3 \vec{r}(\vec{r} \cdot \vec{m}(t))-$ $\left.r^{2} \vec{m}(t)\right] / r^{5}$. This small field is superimposed against the constant external field background $(\vec{B})$. In the above, $\vec{r}$ is the
FIG. 5: A SQUID-based detection scheme. The SQUID monitors the magnetic field produced by the magnetic cluster in one of the junctions.

position relative to the spin and $\vec{m}(t)$ is the magnetic moment of the spin. A ferromagnetic cluster of spin $S=100$ generates a detectable magnetic field $\delta B \sim 10^{-10} \mathrm{~T}$ at a distance of a micron away from the spin. For a SQUID loop of micron dimensions located at that position, the ensuing flux variation $\delta \Phi \sim 10^{-7} \Phi_{0}$ (with $\Phi_{0}=h c / e$ the flux quantum) are within reach of modern SQUIDs. In such a setup, with $T_{1} / T_{0} \sim 0.1$, the typical critical Josephson current is $J_{S}^{(0)} \sim 10 \mu \mathrm{A},|\Delta|=1 \mathrm{meV}$, and $e V \sim 10^{-3}|\Delta|$, we find that the relative corrections $\delta S / S \sim 0.1$. The spin components orthogonal to $\vec{B}$ vary, to $\mathcal{O}\left(T_{1}^{2}\right)$, with Fourier components at frequencies $\left|\omega_{L} \pm \omega_{J}\right|\left(\omega_{L}=g \mu_{B} B\right)$, leading to a observable signal in the magnetic field $\vec{B}+\delta \vec{B}$. For a field $B \sim 200 \mathrm{G}, \omega_{L} \sim 560 \mathrm{MHz}$, and a new side band will appear at $\left|\omega_{L}-\omega_{J}\right|$, whose magnitude may be tuned to $10-100 \mathrm{MHz}$. This measurable frequency is easily distinguished from the Larmor frequency $\omega_{L}$.

The efficiency of this detection scheme may be enhanced by embedding the spin in one of the Josephson junction arms of the SQUID itself. Such a setup is illustrated in Fig. 5 The Josephson junction harboring the spin is employed in both triggering the nutations and, along with the second junction of the SQUID, in the detection of the resulting nutations.

\section{B. Appendix B: Time Ordering Along the Keldysh Contour}

In averaging within the path integral formalism, we immediately attain time ordered averages. In interchanging the order of the spins (if necessary) in the vectorial product upon time ordering within the path integral $C P_{1}$ formulation a change of sign is incurred [31]. We now go over, in some detail, time ordering within the Keldysh framework. As the time ordering is performed along the Keldysh contour, we will denote it by $T_{K}$ as we have done in deriving the effective action of subsection VC.

Consider the third term in Eq. 33. Upon time ordering, we 
find that

$$
\begin{aligned}
\left\langle\vec{S}_{q u}\left(t^{\prime}\right) \times \vec{S}_{c l}(t)\right\rangle_{S}= & \left\langle T_{K}\left[\vec{S}_{q u}\left(t^{\prime}\right) \times \vec{S}_{c l}(t)\right]\right\rangle \\
=\left\langle T_{K}[\right. & \frac{1}{2}\left(\vec{S}_{u p}\left(t^{\prime}\right)-\vec{S}_{\text {down }}\left(t^{\prime}\right)\right) \\
& \left.\left.\times\left(\vec{S}_{\text {up }}(t)+\vec{S}_{\text {down }}(t)\right)\right]\right\rangle .
\end{aligned}
$$

Due to the form of the Keldysh contour (see Fig.(1)), irrespective of the values of $t$ and $t^{\prime}, \vec{S}_{u p}(t)$ always appears before $\vec{S}_{\text {down }}\left(t^{\prime}\right)$. Similarly, for $t>t^{\prime}, \vec{S}_{\text {up }}(t)$ appears after $\vec{S}_{\text {up }}\left(t^{\prime}\right)$ while $\vec{S}_{\text {down }}(t)$ appears before $\vec{S}_{\text {down }}\left(t^{\prime}\right)$. With this information at hand, Eq. 58 leads to

$$
\begin{array}{r}
\left\langle T_{K}\left[\vec{S}_{q u}\left(t^{\prime}\right) \times \vec{S}_{c l}(t)\right]\right\rangle \\
=-\theta\left(t-t^{\prime}\right)\left[\left\langle\vec{S}\left(t^{\prime}\right) \times \vec{S}(t)\right\rangle+\left\langle\vec{S}(t) \times \vec{S}\left(t^{\prime}\right)\right\rangle\right] .
\end{array}
$$

The expectation values on the right are the usual operator expectation values. Here, we disposed of the up/down indices once we took care of time ordering. The up/down labels merely serve as mnemonics for this time ordering along the Keldysh contour.

Similarly, we find that

$$
\begin{array}{r}
\left\langle T_{K}\left[\vec{S}_{c l}\left(t^{\prime}\right) \times \vec{S}_{c l}(t)\right]\right\rangle= \\
\frac{1}{2}\left[\theta\left(t^{\prime}-t\right)\left(\left\langle\vec{S}\left(t^{\prime}\right) \times \vec{S}(t)-\vec{S}(t) \times \vec{S}\left(t^{\prime}\right)\right)\right\rangle\right. \\
\left.+\theta\left(t-t^{\prime}\right)\left(\left\langle\vec{S}(t) \times \vec{S}\left(t^{\prime}\right)-\vec{S}\left(t^{\prime}\right) \times \vec{S}(t)\right)\right\rangle\right] .
\end{array}
$$

By the same token, $\left\langle T_{K}\left[\vec{S}_{q u}(t) \times \vec{S}_{q u}\left(t^{\prime}\right)\right]\right\rangle=0$.

As will become clear shortly, in the solution of Eqs. (33) to order $\mathcal{O}\left(T_{1}^{2}\right)$, we will need the spin-spin expectation values of the usual Larmor problem (i.e. a single spin in a magnetic field sans any supercurrent). Here,

$$
\begin{array}{r}
S_{x}(t)=S_{x}(0) \cos \omega_{L} t+S_{y}(0) \sin \omega_{L} t \\
S_{y}(t)=S_{y}(0) \cos \omega_{L} t-S_{x}(0) \sin \omega_{L} t, \\
S_{z}(t)=S_{z}(0),
\end{array}
$$

with the external magnetic field oriented along the positive $\mathrm{z}$ axis and $\omega_{L}=|\vec{h}|$ the Larmor frequency.

Next, we invoke this solution to compute the various expectation values within the Larmor problem (i.e. to order $\mathcal{O}\left(T_{1}^{0}\right)$ ). We find that

$$
\begin{array}{r}
\left\langle\vec{S}_{q u}\left(t^{\prime}\right) \times \vec{S}_{c l}(t)\right\rangle_{S} \\
=-\theta\left(t-t^{\prime}\right)\left[i \operatorname{Im}\left\{\left\langle\vec{S}\left(t^{\prime}\right) \times \vec{S}(t)\right\rangle\right\}\right. \\
=-i \theta\left(t-t^{\prime}\right)\left\{\left[\left\langle S_{x}(t)\right\rangle\left(1+\cos \omega_{L}\left(t^{\prime}-t\right)\right)\right.\right. \\
\left.+\left\langle S_{y}(t)\right\rangle \sin \omega_{L}\left(t^{\prime}-t\right)\right] \hat{e}_{x} \\
+\left[\left\langle S_{y}(t)\right\rangle\left(1+\cos \omega_{L}\left(t^{\prime}-t\right)\right)\right. \\
\left.-\left\langle S_{x}(t)\right\rangle \sin \omega_{L}\left(t^{\prime}-t\right)\right] \hat{e}_{y} \\
\left.+2\left\langle S_{z}(t)\right\rangle \cos \omega_{L}\left(t^{\prime}-t\right) \hat{e}_{z}\right\}
\end{array}
$$

Similarly, for the $S=1 / 2$ problem,

$$
\begin{aligned}
& \left\langle\vec{S}_{c l}\left(t^{\prime}\right) \times \vec{S}_{c l}(t)\right\rangle_{S} \\
= & \operatorname{Re}\left\{\left\langle\vec{S}\left(t^{\prime}\right) \times \vec{S}(t)\right\rangle\right\} \\
= & \frac{1}{2} \sin \omega_{L}\left(t^{\prime}-t\right) \hat{e}_{z} .
\end{aligned}
$$

In Eqs.6263, we vividly see that upon time ordering along the Keldysh contour, the non-vanishing spin cross products become simply related to the imaginary and real parts of $\left\langle\vec{S}\left(t^{\prime}\right) \times \vec{S}(t)\right\rangle$.

\section{Appendic C: Evaluation of Integrals}

We are now ready for the evaluation of the various integrals $I$ that appear in Eq. 33, to order $\mathcal{O}\left(T_{1}^{2}\right)$.

We start with $\vec{I}_{c l-c l}$. Inserting Eq. 63) and Eq. 30 into Eq. 33 we find upon invoking the relation $\phi(t)=\omega_{J} t$

$$
\begin{array}{r}
\left\langle\vec{I}_{c l-c l}\right\rangle_{S}= \\
4\left|T_{1}\right|^{2} \hat{e}_{z} \int d t^{\prime} j\left(t, t^{\prime}\right) \beta^{R}\left(t, t^{\prime}\right)\left\langle\vec{S}_{c l}\left(t^{\prime}\right) \times \vec{S}_{c l}(t)\right\rangle_{S} \\
=-\left|T_{1}\right|^{2} \hat{e}_{z} \sum_{k, p} \frac{\Delta^{2}}{E_{k} E_{p}} \int d t^{\prime} \theta\left(t-t^{\prime}\right) \cos \omega_{J} \frac{t+t^{\prime}}{2} \\
\times \sin \omega_{L}\left(t-t^{\prime}\right) \sin \left[\left(E_{k}+E_{p}\right)\left(t-t^{\prime}\right)\right] .
\end{array}
$$

Before evaluating Eq.64 exactly, we illustrate what answer is anticipated. The underlying observation of this adiabatic approach is that, as a consequence of $\omega_{L, J} \ll E_{k, p}$ the spin dynamics is far slower than that of electronic processes. Thus, in integrals involving both spin and electronic degrees of freedom, we may regard the spin as nearly stationary and approximate $\vec{S}\left(t^{\prime}\right) \simeq \vec{S}(t)+\left(t^{\prime}-t\right)(d \vec{S} / d t)$. This physically transparent approximation was invoked in [8]. Employing this approximation here we anticipate that $\left\langle\vec{I}_{c l-c l}\right\rangle_{S} \simeq C_{1} \hat{e}_{z} \sin \omega_{L} t$ where $C_{1}$ is, up to trivial prefactors, given by $\int_{0}^{\infty} d x\left[x^{2} \beta^{R}(x)\right]$. Such an anticipation is not far off the mark.

Next, we exactly evaluate Eq.64 by rewriting products of trigonometric functions as sums and consequently employing the identities

$$
\begin{array}{r}
\int_{0}^{\infty} d x \cos a x=\pi \delta(a), \\
\int_{0}^{\infty} d x \sin a x=\pi \delta(a)+\frac{1}{a} .
\end{array}
$$

In the integrals of interest, $x$ assumes the role of $\left(t^{\prime}-t\right)$. As the applied magnetic field and voltage are far lower than electronic energy scales, $\omega_{L, J} \ll E_{k, p}$, we find that all resonances signaled by the delta functions are physically unaccessible and our expressions undergo further simplifications. Retaining the leading order terms in $\mathcal{O}\left(\omega_{L, J} /\left(E_{k}+E_{p}\right)\right)$ we arrive at

$$
\left\langle\vec{I}_{c l-c l}\right\rangle_{S}=-\left|T_{1}\right|^{2} \hat{e}_{z} \sum_{k, p} \frac{\Delta^{2} \omega_{L} \omega_{J}}{E_{k} E_{p}\left(E_{k}+E_{p}\right)^{3}} \sin \omega_{J} t .
$$

Thus, the form anticipated by the adiabatic approximation is correct if $C_{1}=-\left|T_{1}\right|^{2} \sum_{k, p} \frac{\Delta^{2} \omega_{L} \omega_{J}}{E_{k} E_{p}\left(E_{k}+E_{p}\right)^{3}}$. 
Similarly, by inserting Eqs.3162 into Eq. 33 and invoking Eqs. 65, we find

$$
\begin{array}{r}
\left\langle\vec{I}_{q u-c l}\right\rangle_{S}=-\left|T_{1}\right|^{2} \sum_{k, p} \frac{|\Delta|^{2}}{E_{k} E_{p}\left(E_{k}+E_{p}\right)^{2}} \\
\times\left[\left(\left\langle S_{x}(t)\right\rangle \omega_{J} \sin \omega_{J} t-\left\langle S_{y}(t)\right\rangle \omega_{L} \cos \omega_{J} t\right) \hat{e}_{x}\right. \\
+\left(\left\langle S_{y}(t)\right\rangle \omega_{J} \sin \omega_{J} t+\left\langle S_{x}(t)\right\rangle \omega_{L} \cos \omega_{J} t\right) \hat{e}_{y} \\
\left.+\left\langle S_{z}(0)\right\rangle \omega_{J} \sin \omega_{J} t \hat{e}_{z}\right] \\
=C_{2}\left[\omega_{J}\langle\vec{S}(t)\rangle \sin \phi(t)+\omega_{L}\left(\hat{e}_{z} \times\langle\vec{S}(t)\rangle\right) \cos \phi(t)\right],
\end{array}
$$

with the constant $C_{2} \equiv-\left|T_{1}\right|^{2} \sum_{k, p} \frac{|\Delta|^{2}}{E_{k} E_{p}\left(E_{k}+E_{p}\right)^{2}}$ and $\phi(t)=\omega_{J} t$ the superconducting phase difference across the junction. The last line of Eq.67 has a very physically suggestive meaning regarding spin contractions and an effective longitudinal magnetic field- items which we will expand on in Section VF. Our expressions (Eqs. 6667) above are exact to lowest order in $T_{1}$ and the ratios $\left(\omega_{L, J} / E_{k, p}\right)$.

Finally, the integral $\left\langle\vec{I}_{q u-q u}\right\rangle=0$ identically by virtue of a vanishing $\left\langle\vec{S}_{q u}\left(t^{\prime}\right) \times \vec{S}_{q u}(t)\right\rangle_{S}=0$.
[1] J. Koehler et al., Nature 363, 342 (1993); J. Wrachtrup et al., ibid. 363, 244 (1993); Phys. Rev. Lett. 71, 3565 (1993).

[2] H.-A. Engel and D. Loss, Phys. Rev. Lett. 86, 4648 (2001); Phys. Rev. B 65, 195321 (2002).

[3] Y. Manassen et al., Phys. Rev. Lett. 62, 2531 (1989); D. Shachal and Y. Manassen, Phys. Rev. B 46, 4795 (1992); Y. Manassen, J. Magnetic Reson. 126, 133 (1997); Y. Manassen, I. Mukhopadhyay, and N. Ramesh Rao, Phys. Rev. B 61, 16223 (2000).

[4] C. Durkan and M. E. Welland, Appl. Phys. Lett. 80, 458 (2002).

[5] H. Manoharan, Nature 416, 24 (2002); H. Manoharan, C. P. Lutz, and D. Eigler, Nature 403, 512 (2000).

[6] A. V. Balatsky and I. Martin, Quan. Inform. Process. 1, 53 (2002); A. V. Balatsky, Y. Manassen, and R. Salem, Phys. Rev B 66, 195416 (2002).

[7] D. Mozyrsky and I. Martin, Phys. Rev. Lett. 89, 018301 (2002).

[8] J-X. Zhu, Z. Nussinov, A. Shnirman, A. V. Balatsky, Phys. Rev. Lett 92, 107001 (2004)

[9] L. Bulaevskii, M. Hruska, A. Shnirman, D. Smith, Yu. Makhlin, Phys. Rev. Lett. 92 , 177001 (2004).

[10] I. O. Kulik, Sov. Phys. JETP 22, 841 (1966)

[11] V. Ambegaokar and A. Baratoff, Phys. Rev. Lett., 10, 486 (1963)

[12] L. N. Bulaevskii, V. V. Kuzii,and A. A. Sobyanin, JETP Lett. 25, 290 (1977)

[13] L. I. Glazman and K. A. Matveev, JETP Lett., 49, 659 (1989)

[14] W. Wernsdorfer, Adv. Chem. Phys., 118, 99 (2001)

[15] Y. Avishai, A. Golub, and A. D. Zaikin, Phys. Rev. B 63, 134515 (2001).

[16] Yu. Makhlin, Z. Nussinov, A. Shnirman, in preparation

[17] E. Fradkin, Field Theories of Condensed Matter Systems (Addison-Wesley, Redwood City, 1991);

[18] A. Perelomov, "Generalized Coherent States and Their Applications", Springer-Verlang, NY 1986.

[19] For a coherent state oriented along $\hat{\Omega}=$ $(\sin \theta \cos \phi, \sin \theta \sin \phi, \cos \theta)$ on the unit sphere, the corresponding spinor is, up to an arbitrary multiplicative global phase factor $(\exp [i \chi])$, the spinor

$$
z^{*}=\left(\cos \frac{\theta}{2} e^{-i \phi / 2}, \sin \frac{\theta}{2} e^{i \phi / 2}\right) .
$$

While inserting complete coherent spin states at all times steps we invoke

$$
\begin{array}{r}
\left\langle\Omega \mid \Omega^{\prime}\right\rangle=\left(\frac{1+\hat{\Omega} \cdot \hat{\Omega}^{\prime}}{2}\right)^{1 / 2} e^{-i S \psi} \\
\psi=2 \tan ^{-1}\left(\tan \left[\frac{1}{2}\left(\phi-\phi^{\prime}\right)\right] \frac{\cos \left[\frac{1}{2}\left(\theta+\theta^{\prime}\right)\right]}{\cos \left[\frac{1}{2}\left(\theta-\theta^{\prime}\right)\right]}\right)+\chi-\chi^{\prime}
\end{array}
$$

where $\chi$ and $\chi^{\prime}$ depend on gauge convention, with $|\Omega\rangle$ and $\left|\Omega^{\prime}\right\rangle$ spin coherent states at two different times. In the limit of small deviations $\left(\phi^{\prime}-\phi\right)$ and $\left(\theta^{\prime}-\theta\right)$, the above argument reduces to $d \psi=[(d \phi / d t) \cos \theta] d t$ which is simply the area elements swept by $\hat{\Omega}(t)$ in an infinitesimal time increment $d t$. Within the path integral formulation we insert complete sets of coherent states at different times. We see from the internal product above that within the spin coherent basis the path integral attains a Berry phase which is simply the area swept by the spin its cyclic evolution. (It is cyclic due to the requirement of taking a trace within the partition function).

An explicit evaluation with the spinor of Eq. 68 reveals that

$$
\mathcal{L}_{\text {Berry }}=z_{a}^{*}\left(i \partial_{t}\right) z_{a}
$$

is none other than $[(i / 2)(d \phi / d t) \cos \theta]$. Upon integration over time this leads, up to trivial additive constants, to $(i / 2)$ times the area spanned by the spin.

[20] A. Das, "Finite Temperature Field Theory", World Scientific, 1997

[21] A. Kamenev, cond-mat/0109316

[22] J-X. Zhu, A. V. Balatsky, Phys. Rev. B 67, 174505 (2003)

[23] V. Ambegaokar, U. Eckern, G. Schön, Phys. Rev. Lett. 48, 1745 (1982).

[24] A. I. Larkin, Y. N. Ovchinnikov, Phys. Rev. B 28, 6281 (1983).

[25] U. Eckern, G. Schön, V. Ambegaokar, Phys. Rev. B 30, 6419 (1984).

[26] Z. Nussinov and A. Shnirman, unpublished

[27] S. Shapiro, Phys. Rev. Lett. 11, 80 (1963)

[28] F. J. Dyson, Phys. Rev. 102, 1230 (1956), S. Maleev, Zh. Eksp. Teor. Fiz., 33, 1010 (1957)

[29] Z. Nussinov, A. Shnirman, D. P. Arovas, A. V. Balatsky, J-X. Zhu, In preparation

[30] A. Shnirman et al., Fizika Nizkikh Temperatur 7/8, 30 (2004)

[31] The illustration of this change of sign upon exchanging the order of times within the path integral average is straightforward.

$$
\begin{array}{r}
\int D z D z^{*} \delta\left(|z|^{2}-1\right) \epsilon_{i j k} z_{a}^{*}\left(t_{2}\right) \sigma_{a b}^{i} z_{b}\left(t_{2}\right) z_{c}^{*}\left(t_{1}\right) \sigma_{c d}^{j} z_{d}\left(t_{1}\right) e^{i S}= \\
-\int D z D z^{*} \delta\left(|z|^{2}-1\right) \epsilon_{i j k} z_{a^{\prime}}^{*}\left(t_{1}\right) \sigma_{a^{\prime} b^{\prime}}^{i} z_{b^{\prime}}\left(t_{1}\right) z_{c^{\prime}}^{*}\left(t_{2}\right) \sigma_{c^{\prime} d^{\prime}}^{j} z_{d^{\prime}}\left(t_{2}\right) e^{i S} .
\end{array}
$$

This can be seen by making an identification of summation indices, $a^{\prime}=c, b^{\prime}=d, c^{\prime}=a, d^{\prime}=b$, and invoking $\epsilon_{i j k}=-\epsilon_{j i k}$. 